\title{
Perturbed eigenvalue problems for the Robin $p$-Laplacian plus an indefinite potential
}

\section{Calogero Vetro ${ }^{1}$}

Received: 17 September 2019 / Revised: 17 September 2019 / Accepted: 18 October 2020 /

Published online: 1 November 2020

(c) The Author(s) 2020

\section{Abstract}

We consider a parametric nonlinear Robin problem driven by the negative $p$-Laplacian plus an indefinite potential. The equation can be thought as a perturbation of the usual eigenvalue problem. We consider the case where the perturbation $f(z, \cdot)$ is $(p-1)$ sublinear and then the case where it is $(p-1)$-superlinear but without satisfying the Ambrosetti-Rabinowitz condition. We establish existence and uniqueness or multiplicity of positive solutions for certain admissible range for the parameter $\lambda \in \mathbb{R}$ which we specify exactly in terms of principal eigenvalue of the differential operator.

Keywords Positive solutions - Sublinear and superlinear perturbation · Nonlinear Picone's identity · Nonlinear maximum principle · Nonlinear regularity · Indefinite potential $\cdot$ Minimal positive solution $\cdot$ Uniqueness

Mathematics Subject Classification Primary: 35J20; Secondary: 35J60

\section{Introduction}

Let $\Omega \subseteq \mathbb{R}^{N}$ be a bounded domain with a $C^{2}$-boundary $\partial \Omega$. In this paper we study the following nonlinear parametric Robin problem

$$
\begin{cases}-\Delta_{p} u(z)+\xi(z)|u(z)|^{p-2} u(z)=\lambda|u(z)|^{p-2} u(z)+f(z, u(z)) & \text { in } \Omega, \\ \frac{\partial u}{\partial n_{p}}+\beta(z)|u|^{p-2} u=0 & \text { on } \partial \Omega, u \geq 0, \lambda \in \mathbb{R} .\end{cases}
$$

In this problem $\Delta_{p}$ denotes the $p$-Laplace differential operator defined by

$$
\Delta_{p} u=\operatorname{div}\left(|\nabla u|^{p-2} \nabla u\right) \quad \text { for all } u \in W^{1, p}(\Omega) \quad(1<p<+\infty) .
$$

Calogero Vetro

calogero.vetro@unipa.it

1 Department of Mathematics and Computer Science, University of Palermo, Via Archirafi 34, 90123

Palermo, Italy 
Also $\xi(\cdot) \in L^{\infty}(\Omega)$ is an indefinite (that is, sign changing) potential function, $\lambda \in \mathbb{R}$ is a parameter and $f(z, x)$ is a Carathéodory perturbation function (that is, for all $x \in \mathbb{R}, z \rightarrow f(z, x)$ is measurable and for a.a. $z \in \Omega, x \rightarrow f(z, x)$ is continuous). In the boundary condition $\frac{\partial u}{\partial n_{p}}$ denotes the generalized normal derivative defined by

$$
\frac{\partial u}{\partial n_{p}}=|\nabla u|^{p-2}(\nabla u, n)_{\mathbb{R}^{N}}=|\nabla u|^{p-2} \frac{\partial u}{\partial n} \text { for all } u \in W^{1, p}(\Omega),
$$

with $n(\cdot)$ being the outward unit normal on $\partial \Omega$. This kind of generalized normal derivative is dictated by the nonlinear Green's identity (see, for example, GasińskiPapageorgiou [8] (p. 211)). The boundary weight term $\beta \in C^{0, \alpha}(\partial \Omega)(0<\alpha<1)$ and $\beta(z) \geq 0$ for all $z \in \partial \Omega$.

Problem $\left(P_{\lambda}\right)$ can be viewed as a perturbation of the usual eigenvalue problem for the Robin $p$-Laplacian plus an indefinite potential. We look for positive solutions and we consider two distinct cases depending on the growth of the perturbation $f(z, \cdot)$ near $+\infty$ :

- $f(z, \cdot)$ is $(p-1)$-sublinear.

- $f(z, \cdot)$ is $(p-1)$-superlinear.

Let $\widehat{\lambda}_{1} \in \mathbb{R}$ be the principal eigenvalue of the differential operator $u \rightarrow-\Delta_{p} u+$ $\xi(z)|u|^{p-2} u$ with Robin boundary condition. In the first case $((p-1)$-sublinear perturbation), we show that for all $\lambda \geq \widehat{\lambda}_{1}$, problem $\left(P_{\lambda}\right)$ has no positive solution, while for $\lambda<\widehat{\lambda}_{1}$, problem $\left(P_{\lambda}\right)$ has at least one positive solution. Moreover, this positive solution is unique, if we impose a monotonicity condition on the quotient $x \rightarrow \frac{f(z, x)}{x^{p-1}}$ for $x>0$. In the second case $((p-1)$-superlinear perturbation), the situation changes and uniqueness of the positive solution fails. In fact the problem exhibits a kind of bifurcation phenomenon. Namely, for $\lambda \geq \widehat{\lambda}_{1}$ problem $\left(P_{\lambda}\right)$ has no positive solution, while for $\lambda<\widehat{\lambda}_{1}$ problem $\left(P_{\lambda}\right)$ has at least two positive solutions. Finally for both situations, we establish the existence of minimal positive solutions. Our work here extends to the $p$-Laplacian that of Papageorgiou-Rădulescu-Repovš [20]. Eigenvalue problems for the $p$-Laplacian plus an indefinite potential were studied by Papageorgiou-Rădulescu [18] (semilinear problems (that is, $p=2$ ) with Robin boundary condition) and by Mugnai-Papageorgiou [16] (nonlinear problems with Neumann boundary condition (that is, $\beta \equiv 0)$ ). Both works deal with nonparametric problems and prove existence and multiplicity results under resonance conditions. We also mention the works of $\mathrm{Hu}-$ Papageorgiou [10-12]. In [11] the authors treat superdiffusive logistic equation with Robin boundary condition, while in $[10,12]$, they deal with equations driven by a nonhomogeneous differential operator.

\section{Auxiliary results}

In this section we present some auxiliary results and notions which we will need in the sequel. 
First we deal with the following eigenvalue problem:

$$
\begin{cases}-\Delta_{p} u(z)+\xi(z)|u(z)|^{p-2} u(z)=\widehat{\lambda}|u(z)|^{p-2} u(z) & \text { in } \Omega \\ \frac{\partial u}{\partial n_{p}}+\beta(z)|u|^{p-2} u=0 & \text { on } \partial \Omega .\end{cases}
$$

Our hypotheses on the functions $\xi(\cdot)$ and $\beta(\cdot)$ are the following:

$H(\xi): \xi \in L^{\infty}(\Omega)$.

$H(\beta)_{1}: \beta \in C^{0, \alpha}(\partial \Omega)$ with $\alpha \in(0,1)$ and $\beta(z) \geq 0$ for all $z \in \partial \Omega$.

In addition to the Sobolev space $W^{1, p}(\Omega)$, we will also use the Banach space $C^{1}(\bar{\Omega})$ which is an ordered Banach space with positive cone $C_{+}=\left\{u \in C^{1}(\bar{\Omega})\right.$ : $u(z) \geq 0$ for all $z \in \bar{\Omega}\}$. This cone has a nonempty interior given by

$$
D_{+}=\left\{u \in C_{+}: u(z)>0 \text { for all } z \in \bar{\Omega}\right\} .
$$

Also on $\partial \Omega$ we consider the $(N-1)$-dimensional Hausdorff (surface) measure $\sigma(\cdot)$. With this measure on $\partial \Omega$, we can define the Lebesgue spaces $L^{\tau}(\partial \Omega) 1 \leq \tau \leq+\infty$. We know that there exists a unique continuous linear map $\gamma_{0}: W^{1, p}(\Omega) \rightarrow L^{p}(\partial \Omega)$ known as the "trace map" s.t. $\gamma_{0}(u)=\left.u\right|_{\partial \Omega}$ for all $u \in W^{1, p}(\Omega) \cap C(\bar{\Omega})$. So, we understand the trace map as representing the "boundary values" of a Sobolev function $u \in W^{1, p}(\Omega)$. We know that $\gamma_{0}$ is compact into $L^{\tau}(\partial \Omega)$ for all $\tau \in\left[1, \frac{(N-1) p}{N-p}\right)$ when $p<N$ and into $L^{\tau}(\partial \Omega)$ for all $\tau \in[1,+\infty)$ when $p \geq N$. Moreover, we have

$$
\operatorname{im} \gamma_{0}=W^{\frac{1}{p^{\prime}}, p}(\partial \Omega) \quad\left(\frac{1}{p}+\frac{1}{p^{\prime}}=1\right) \text { and } \operatorname{ker} \gamma_{0}=W_{0}^{1, p}(\Omega) .
$$

In the sequel for the sake of notational simplicity we drop the use of the trace map $\gamma_{0}$. It is understood that all restrictions of Sobolev functions on $\partial \Omega$ are taken in the sense of traces.

In what follows by $\vartheta: W^{1, p}(\Omega) \rightarrow \mathbb{R}$ we denote the $C^{1}$-functional defined by

$$
\vartheta(u)=\|\nabla u\|_{p}^{p}+\int_{\Omega} \xi(z)|u|^{p} d z+\int_{\partial \Omega} \beta(z)|u|^{p} d \sigma \quad \text { for all } u \in W^{1, p}(\Omega) .
$$

From Fragnelli-Mugnai-Papageorgiou [7], we have the following proposition concerning problem (1) (see also Mugnai-Papageorgiou [16] and PapageorgiouRădulescu [18] where special cases of (1) are investigated).

Proposition 1 If hypotheses $H(\xi), H(\beta)_{1}$ hold, then problem (1) admits a smallest eigenvalue $\widehat{\lambda}_{1} \in \mathbb{R}$ s.t.

$$
\widehat{\lambda}_{1}=\inf \left[\frac{\vartheta(u)}{\|u\|_{p}^{p}}: u \in W^{1, p}(\Omega), u \neq 0\right]
$$


- $\widehat{\lambda}_{1}$ is isolated and simple.

- The infimum in (2) is realized on the one-dimensional eigenspace of $\widehat{\lambda}_{1}$; the elements of this eigenspace do not change sign and if $\widehat{u}_{1}$ denotes the positive, $L^{p}$-normalized (that is, $\left\|\widehat{u}_{1}\right\|_{p}=1$ ) eigenfunction, then $\widehat{u}_{1} \in D_{+}$.

- If $\widehat{\lambda}>\widehat{\lambda}_{1}$ is another eigenvalue and $\widehat{u} \in W^{1, p}(\Omega)$ a corresponding eigenfunction, then $\widehat{u} \in C^{1}(\bar{\Omega})$ is nodal (that is, sign changing).

As a consequence of these properties, we have the following useful lemma.

Lemma 1 If hypotheses $H(\xi), H(\beta)_{1}$ hold, $\eta \in L^{\infty}(\Omega), \eta(z) \leq \widehat{\lambda}_{1}$ for a.a. $z \in \Omega$ and the inequality is strict on a set of positive measure, then there exists $\widehat{c}>0$ s.t.

$$
\widehat{c}\|u\|^{p} \leq \vartheta(u)-\int_{\Omega} \eta(z)|u|^{p} d z \text { for all } u \in W^{1, p}(\Omega) .
$$

Proof Let $\zeta: W^{1, p}(\Omega) \rightarrow \mathbb{R}$ be the $C^{1}$-functional defined by

$$
\zeta(u)=\vartheta(u)-\int_{\Omega} \eta(z)|u|^{p} d z \quad \text { for all } u \in W^{1, p}(\Omega) .
$$

From (2) we have $\zeta \geq 0$. Suppose that the claim of the lemma is not true. Then we can find $\left\{u_{n}\right\}_{n \geq 1} \subseteq W^{1, p}(\Omega)$ s.t.

$$
\zeta\left(u_{n}\right) \downarrow 0 \text { as } n \rightarrow+\infty .
$$

The $p$-homogeneity of $\zeta(\cdot)$ implies that we may assume that $\left\|u_{n}\right\|_{p}=1$ for all $n \in \mathbb{N}$. Then clearly $\left\{u_{n}\right\}_{n \geq 1} \subseteq W^{1, p}(\Omega)$ is bounded (see hypotheses $\left.H(\xi), H(\beta)_{1}\right)$ and so we may assume that

$$
u_{n} \stackrel{w}{\rightarrow} u \text { in } W^{1, p}(\Omega) \text { and } u_{n} \rightarrow u \text { in } L^{p}(\Omega) \text { and in } L^{p}(\partial \Omega),\|u\|_{p}=1 .
$$

From (3) and (4), we obtain

$$
\begin{aligned}
& \vartheta(u) \leq \int_{\Omega} \eta(z)|u|^{p} d z \leq \widehat{\lambda}_{1}\|u\|_{p}^{p}=\widehat{\lambda}_{1} \\
& \quad \Rightarrow \vartheta(u)=\widehat{\lambda}_{1} \quad(\text { see }(2)), \\
& \left.\quad \Rightarrow u=\mu \widehat{u}_{1} \quad \text { with } \mu \neq 0 \text { (see Proposition } 1\right) .
\end{aligned}
$$

To fix things we assume that $\mu>0$ (the reasoning is the same if $\mu<0$ ). Then from (5) and since $u=\mu \widehat{u}_{1} \in D_{+}$, we have

$$
\vartheta(u)<\widehat{\lambda}_{1}
$$

wich contradicts (2). 
Let $A: W^{1, p}(\Omega) \rightarrow W^{1, p}(\Omega)^{*}$ be the nonlinear map defined by

$$
\langle A(u), h\rangle=\int_{\Omega}|\nabla u|^{p-2}(\nabla u, \nabla h)_{\mathbb{R}^{N}} d z \text { for all } u, h \in W^{1, p}(\Omega) .
$$

From Motreanu-Motreanu-Papageorgiou [14] (p. 40), we have the following result concerning this map.

Proposition 2 The map A(·) is bounded (that is, maps bounded sets to bounded sets), monotone, continuous (hence maximal monotone too) and of type $(S)_{+}$, that is, if $u_{n} \stackrel{w}{\rightarrow} u$ in $W^{1, p}(\Omega)$ and $\limsup _{n \rightarrow+\infty}\left\langle A\left(u_{n}\right), u_{n}-u\right\rangle \leq 0$, then $u_{n} \rightarrow u$ in $W^{1, p}(\Omega)$.

Recall that if $X$ is a Banach space and $\varphi \in C^{1}(X, \mathbb{R})$, then we say that $\varphi$ satisfies the Cerami condition (the $C$-condition for short), if the following is true:

"Every sequence $\left\{u_{n}\right\}_{n \geq 1} \subseteq X$ s.t. $\left\{\varphi\left(u_{n}\right)\right\}_{n \geq 1} \subseteq \mathbb{R}$ is bounded and (1+ $\left.\left\|u_{n}\right\|\right) \varphi^{\prime}\left(u_{n}\right) \rightarrow 0$ in $X^{*}$ as $n \rightarrow+\infty$, admits a strongly convergent subsequence".

Let $f_{0}: \Omega \times \mathbb{R} \rightarrow \mathbb{R}$ be a Carathéodory function s.t.

$$
\left|f_{0}(z, x)\right| \leq a(z)\left(1+|x|^{p^{*}-1}\right) \text { for a.a. } z \in \Omega \text {, all } x \in \mathbb{R} \text {, }
$$

with $a \in L^{\infty}(\Omega)_{+}$and $p^{*}=\left\{\begin{array}{ll}\frac{N p}{N-p} & \text { if } p<N \\ +\infty & \text { if } N \leq p\end{array}\right.$ (the critical Sobolev exponent). Let $F_{0}(z, x)=\int_{0}^{x} f_{0}(z, s) d s$ and consider the $C^{1}$-functional $\varphi_{0}: W^{1, p}(\Omega) \rightarrow \mathbb{R}$ defined by

$$
\varphi_{0}(u)=\frac{1}{p} \vartheta(u)-\int_{\Omega} F_{0}(z, u) d z \text { for all } u \in W^{1, p}(\Omega) .
$$

From Papageorgiou-Rădulescu [17], we have the following result relating local minimizers of $\varphi_{0}$ and which is an outgrowth of the nonlinear regularity theory. The first such result was proved by Brezis-Nirenberg [4] for $p=2$ and the space $H_{0}^{1}(\Omega)$.

Proposition 3 If $u_{0} \in W^{1, p}(\Omega)$ is a local $C^{1}(\bar{\Omega})$-minimizer of $\varphi_{0}$, that is, there exists $\delta_{1}>0$ s.t.

$$
\varphi_{0}\left(u_{0}\right) \leq \varphi_{0}\left(u_{0}+h\right) \text { for all } h \in C^{1}(\bar{\Omega}),\|h\|_{C^{1}(\bar{\Omega})} \leq \delta_{1},
$$

then $u_{0} \in C^{1, \tau}(\bar{\Omega})$ with $\tau \in(0,1)$ and it is also a local $W^{1, p}(\Omega)$-minimizer of $\varphi_{0}$, that is, there exists $\delta_{2}>0$ s.t.

$$
\varphi_{0}\left(u_{0}\right) \leq \varphi_{0}\left(u_{0}+h\right) \text { for all } h \in W^{1, p}(\Omega),\|h\| \leq \delta_{2} .
$$

To make good use of this result, we need a strong comparison principle. In this direction we have the following proposition which is a special case of a more general 
result due to Fragnelli-Mugnai-Papageorgiou [6]. Given $h_{1}, h_{2} \in L^{\infty}(\Omega)$, we say that $h_{1} \prec h_{2}$ if and only if for every $K \subseteq \Omega$ compact, there exists $\varepsilon=\varepsilon(K)>0$ s.t.

$$
h_{1}(z)+\varepsilon \leq h_{2}(z) \text { for a.a. } z \in K \text {. }
$$

Note that if $h_{1}, h_{2} \in C(\Omega)$ and $h_{1}(z)<h_{2}(z)$ for all $z \in \Omega$, then $h_{1} \prec h_{2}$.

Proposition 4 If $\xi, h_{1}, h_{2} \in L^{\infty}(\Omega), h_{1} \prec h_{2}, u \in C^{1}(\bar{\Omega}) \backslash\{0\}, v \in D_{+}$and they satisfy

$$
\begin{aligned}
& -\Delta_{p} u(z)+\xi(z)|u(z)|^{p-2} u(z)=h_{1}(z) \text { for a.a. } z \in \Omega, \\
& -\Delta_{p} v(z)+\xi(z) v(z)^{p-1}=h_{2}(z) \text { for a.a. } z \in \Omega, \frac{\partial v}{\partial n}<0 \text { on } \partial \Omega,
\end{aligned}
$$

then $(v-u)(z)>0$, for all $z \in \Omega$ and $\left.\frac{\partial(v-u)}{\partial n}\right|_{D_{0}}<0$ where $D_{0}=\{z \in \partial \Omega$ : $v(z)=u(z)\}$.

Remark 1 If in $C^{1}(\bar{\Omega})$ we introduce the order cone

$$
\widehat{C}_{+}=\left\{y \in C^{1}(\bar{\Omega}): y(z) \geq 0 \text { for all } z \in \bar{\Omega}, \frac{\partial y}{\partial n} \leq 0 \text { on } D_{0}\right\}
$$

then the above proposition says that $v-u \in \operatorname{int} \widehat{C}_{+}$. If $D_{0}=\emptyset$, then $\widehat{C}_{+}=C_{+}$.

For problem $\left(P_{\lambda}\right)$, we introduce the following two sets:

$$
\begin{aligned}
\mathcal{L} & =\left\{\lambda \in \mathbb{R}: \text { problem }\left(P_{\lambda}\right) \text { admits a positive solution }\right\}, \\
S(\lambda) & =\left\{\text { set of positive solutions for problem }\left(P_{\lambda}\right)\right\}
\end{aligned}
$$

For the set $S(\lambda)$ we have the following general result.

Proposition 5 If hypotheses $H(\xi), H(\beta)_{1}$ hold and $f: \Omega \times \mathbb{R} \rightarrow \mathbb{R}$ is a Carathéodory function s.t.for a.a. $z \in \Omega, f(z, 0)=0, f(z, x) \geq 0$ for all $x>0, f(z, x)=0$ for all $x<0$ and $f(z, x) \leq a(z)\left(1+x^{p^{*}-1}\right)$ for a.a. $z \in \Omega$, all $x \geq 0$, with $a \in L^{\infty}(\Omega)_{+}$, then $S(\lambda) \subseteq D_{+}$(possibly empty).

Proof Suppose that $u \in S(\lambda)$. Then

$$
\begin{cases}-\Delta_{p} u(z)+\xi(z) u(z)^{p-1}=\lambda u(z)^{p-1}+f(z, u(z)) & \text { for a.a. } z \in \Omega \\ \frac{\partial u}{\partial n_{p}}+\beta(z) u^{p-1}=0 & \text { on } \partial \Omega\end{cases}
$$

(see Papageorgiou-Rădulescu [17]). From (6) and Papageorgiou-Rădulescu [19] we have $u \in L^{\infty}(\Omega)$. Then Theorem 2 of Lieberman [13] implies that $u \in C_{+} \backslash\{0\}$. From (6) and since $f \geq 0$, we have

$$
\Delta_{p} u(z) \leq\left(\|\xi\|_{\infty}+|\lambda|\right) u(z)^{p-1} \text { for a.a. } z \in \Omega,
$$


$\Rightarrow u \in D_{+} \quad$ (by the nonlinear strong maximum principle (see [8] (p. 738)).

Proposition 6 If hypotheses $H(\xi), H(\beta)_{1}$ hold, $f: \Omega \times \mathbb{R} \rightarrow \mathbb{R}$ is a Carathéodory function s.t. for a.a. $z \in \Omega f(z, 0)=0, f(z, x)>0$ for all $x>0, f(z, x) \leq$ $a(z)\left(1+x^{p^{*}-1}\right)$ for a.a. $z \in \Omega$, all $x \geq 0$, with $a \in L^{\infty}(\Omega)_{+}$and $\lambda \geq \widehat{\lambda}_{1}$, then $S(\lambda)=\emptyset$.

Proof Arguing by contradiction, suppose that $S(\lambda) \neq \varnothing$ and let $u \in S(\lambda)$. From Proposition 5 we know that $u \in D_{+}$. Also, let $\widehat{u}_{1} \in D_{+}$be the principal eigenfunction from Proposition 5. Consider the function

$$
R\left(\widehat{u}_{1}, u\right)(z)=\left|\nabla \widehat{u}_{1}(z)\right|^{p}-|\nabla u(z)|^{p-2}\left(\nabla u(z), \nabla\left(\frac{\widehat{u}_{1}^{p}}{u^{p-1}}\right)(z)\right)_{\mathbb{R}^{N}}
$$

From the nonlinear Picone's identity of Allegretto-Huang [2] (see also MotreanuMotreanu-Papageorgiou [14] (p. 255)), we have

$$
0 \leq R\left(\widehat{u}_{1}, u\right)(z) \text { for a.a. } z \in \Omega \text {. }
$$

Then we have

$$
\begin{aligned}
0 & \leq \int_{\Omega} R\left(\widehat{u}_{1}, u\right) d z \\
& =\left\|\nabla \widehat{u}_{1}\right\|_{p}^{p}-\int_{\Omega}|\nabla u|^{p-2}\left(\nabla u, \nabla\left(\frac{\widehat{u}_{1}^{p}}{u^{p-1}}\right)\right)_{\mathbb{R}^{N}} d z \\
& =\left\|\nabla \widehat{u}_{1}\right\|_{p}^{p}-\int_{\Omega}\left(-\Delta_{p} u\right) \frac{\widehat{u}_{1}^{p}}{u^{p-1}} d z+\int_{\partial \Omega} \beta(z) \widehat{u}_{1}^{p} d \sigma
\end{aligned}
$$

(by the nonlinear Green's identity, see Gasiński-Papageorgiou [8] (p. 211))

$$
\begin{aligned}
& =\left\|\nabla \widehat{u}_{1}\right\|_{p}^{p}-\int_{\Omega}(\lambda-\xi(z)) \widehat{u}_{1}^{p} d z-\int_{\Omega} f(z, u) \frac{\widehat{u}_{1}^{p}}{u^{p-1}} d z+\int_{\partial \Omega} \beta(z) \widehat{u}_{1}^{p} d \sigma \\
& <\vartheta\left(\widehat{u}_{1}\right)-\lambda \quad\left(\text { since } f(z, u(z)) \frac{\widehat{u}_{1}^{p}}{u^{p-1}}(z)>0 \text { for a.a. } z \in \Omega \text { and }\left\|\widehat{u}_{1}\right\|_{p}=1\right) \\
& =\widehat{\lambda}_{1}-\lambda \leq 0,
\end{aligned}
$$

a contradiction. Therefore $S(\lambda)=\emptyset$ for all $\lambda \geq \widehat{\lambda}_{1}$.

\section{$3(p-1)$-sublinear perturbation}

In this section, we deal with the case of a $(p-1)$-sublinear perturbation $f(z, \cdot)$. $H_{1}: f: \Omega \times \mathbb{R} \rightarrow \mathbb{R}$ is a Carathéodory function s.t. for a.a. $z \in \Omega, f(z, 0)=0$, $f(z, x)>0$ for all $x>0$ and 
(i) for every $\rho>0$, there exists $a_{\rho} \in L^{\infty}(\Omega)_{+}$s.t. $f(z, x) \leq a_{\rho}(z)$ for a.a. $z \in \Omega$, all $x \in[0, \rho]$

(ii) $\lim _{x \rightarrow+\infty} \frac{f(z, x)}{x^{p-1}}=0$ uniformly for a.a. $z \in \Omega$;

(iii) there exist $\delta>0, q \in(1, p)$ and $c_{1}>0$ s.t.

$$
c_{1} x^{q-1} \leq f(z, x) \text { for a.a. } z \in \Omega \text {, all } x \in[0, \delta] .
$$

Remark 2 Since we are looking for positive solutions and the above hypotheses concern the positive semiaxis $\mathbb{R}_{+}=[0,+\infty)$, without any loss of generality we may assume that $f(z, x)=0$ for a.a. $z \in \Omega$, all $x<0$. Hypothesis $H_{1}$ (ii) says that for a.a. $z \in \Omega$ the perturbation $f(z, \cdot)$ is $(p-1)$-sublinear near $+\infty$. Finally hypothesis $H_{1}$ (iii) implies the presence of a concave term near $0^{+}$.

Example 1 The following functions satisfy hypotheses $H_{1}$. For the sake of simplicity we drop the $z$-dependence.

$$
\begin{aligned}
& f_{1}(x)=x^{q-1} \text { for all } x \geq 0 \text { with } 1<q<p, \\
& f_{2}(x)=\left\{\begin{array}{ll}
x^{q-1}-x^{\tau-1} & \text { if } x \in[0,1], \\
\ln x^{p-1} & \text { if } 1<x,
\end{array} \text { with } 1<q<p, 1<q<\tau .\right.
\end{aligned}
$$

Proposition 7 If hypotheses $H(\xi), H(\beta)_{1}, H_{1}$ hold and $\lambda<\widehat{\lambda}_{1}$, then $S(\lambda) \neq \varnothing$ and so $\mathcal{L}=\left(-\infty, \widehat{\lambda}_{1}\right)$.

Proof Let $\eta>\|\xi\|_{\infty}$ and consider the following Carathéodory function

$$
g_{\lambda}(z, x)= \begin{cases}0 & \text { if } x \leq 0 \\ (\lambda+\eta) x^{p-1}+f(z, x) & \text { if } 0<x\end{cases}
$$

We set $G_{\lambda}(z, x)=\int_{0}^{x} g_{\lambda}(z, s) d s$ and consider the $C^{1}$-functional $\varphi_{\lambda}: W^{1, p}(\Omega) \rightarrow$ $\mathbb{R}$ defined by

$$
\varphi_{\lambda}(u)=\frac{1}{p} \vartheta(u)+\frac{\eta}{p}\|u\|_{p}^{p}-\int_{\Omega} G_{\lambda}(z, u) d z \text { for all } u \in W^{1, p}(\Omega) .
$$

Hypotheses $H_{1}(\mathrm{i})$, (ii) imply that given $\varepsilon>0$, we can find $c_{2}=c_{2}(\varepsilon)>0$ s.t.

$$
F(z, x) \leq \frac{\varepsilon}{p} x^{p}+c_{2} \text { for a.a. } z \in \Omega, \text { all } x \geq 0
$$

Then for all $u \in W^{1, p}(\Omega)$ we have

$$
\begin{aligned}
\varphi_{\lambda}(u) & \geq \frac{1}{p} \vartheta(u)+\frac{\eta}{p}\left\|u^{-}\right\|_{p}^{p}-\frac{\lambda+\varepsilon}{p}\left\|u^{+}\right\|_{p}^{p}-c_{2}|\Omega|_{N} \quad \text { (see (7), (8)) } \\
& \geq \frac{1}{p} \vartheta(u)-\frac{\lambda+\varepsilon}{p}\|u\|_{p}^{p}-c_{2}|\Omega|_{N} .
\end{aligned}
$$


Here by $|\cdot|_{N}$ we denote the Lebesgue measure on $\mathbb{R}^{N}$. Choosing $\varepsilon \in\left(0, \widehat{\lambda}_{1}-\lambda\right)$ (recall that $\lambda<\widehat{\lambda}_{1}$ ), from (9) and Lemma 1, we have

$$
\begin{aligned}
\varphi_{\lambda}(u) & \geq c_{3}\|u\|^{p}-c_{2}|\Omega|_{N} \quad \text { for some } c_{3}>0, \text { all } u \in W^{1, p}(\Omega), \\
& \Rightarrow \varphi_{\lambda}(\cdot) \text { is coercive. }
\end{aligned}
$$

Using the Sobolev embedding theorem and the compactness of the trace operator, we see that

$\varphi_{\lambda}(\cdot)$ is sequentially weakly lower semicontinuous.

Then invoking the Weierstrass-Tonelli theorem, we can find $u_{\lambda} \in W^{1, p}(\Omega)$ s.t.

$$
\varphi_{\lambda}\left(u_{\lambda}\right)=\inf \left[\varphi_{\lambda}(u): u \in W^{1, p}(\Omega)\right]
$$

Let $t \in(0,1)$ be small s.t.

$$
t \widehat{u}_{1}(z) \in(0, \delta] \text { for all } z \in \bar{\Omega} \quad\left(\text { recall } \widehat{u}_{1} \in D_{+}\right)
$$

Here $\delta>0$ is as in hypothesis $H_{1}$ (iii). Then we have

$$
\begin{aligned}
\varphi_{\lambda}\left(t \widehat{u}_{1}\right) & \leq \frac{t^{p}}{p} \vartheta\left(\widehat{u}_{1}\right)-\frac{t^{p}}{p} \lambda-\frac{t^{q}}{q} c_{1}\left\|\widehat{u}_{1}\right\|_{q}^{q} \quad\left(\text { see }(7) \text { and hypothesis } H_{1}(\mathrm{iii})\right) \\
& =\frac{t^{p}}{p}\left[\widehat{\lambda}_{1}-\lambda\right]-\frac{t^{q}}{q} c_{1}\left\|\widehat{u}_{1}\right\|_{q}^{q} \quad\left(\text { see Proposition } 1 \text { and recall that }\left\|\widehat{u}_{1}\right\|_{p}=1\right) \\
& =\frac{t^{p}}{p} c_{4}-\frac{t^{q}}{q} c_{5} \quad \text { with } c_{4}=\widehat{\lambda}_{1}-\lambda>0, c_{5}=c_{1}\left\|\widehat{u}_{1}\right\|_{q}^{q}>0 .
\end{aligned}
$$

Since $t \in(0,1)$ and $q<p$, by choosing $t \in(0,1)$ even smaller if necessary, we have

$$
\begin{aligned}
& \varphi_{\lambda}\left(t \widehat{u}_{1}\right)<0 \\
& \quad \Rightarrow \varphi_{\lambda}\left(u_{\lambda}\right)<0=\varphi_{\lambda}(0) \quad(\text { see }(10)), \\
& \quad \Rightarrow u_{\lambda} \neq 0
\end{aligned}
$$

From (10), we have

$$
\begin{aligned}
& \varphi_{\lambda}^{\prime}\left(u_{\lambda}\right)=0, \\
& \quad \Rightarrow\left\langle A\left(u_{\lambda}\right), h\right\rangle+\int_{\Omega}(\xi(z)+\eta)\left|u_{\lambda}\right|^{p-2} u_{\lambda} h d z+\int_{\partial \Omega} \beta(z)\left|u_{\lambda}\right|^{p-2} u_{\lambda} h d \sigma \\
& \quad=\int_{\Omega}\left[(\lambda+\eta)\left(u_{\lambda}^{+}\right)^{p-1}+f\left(z, u_{\lambda}^{+}\right)\right] h d z \text { for all } h \in W^{1, p}(\Omega) .
\end{aligned}
$$


In (11) we choose $h=-u_{\lambda}^{-} \in W^{1, p}(\Omega)$. Then

$$
\begin{aligned}
& \vartheta\left(u_{\lambda}^{-}\right)+\eta\left\|u_{\lambda}^{-}\right\|_{p}^{p}=0, \\
& \quad \Rightarrow c_{6}\left\|u_{\lambda}^{-}\right\|^{p} \leq 0 \text { for some } c_{6}>0 \\
& \quad\left(\text { recall that } \eta>\|\xi\|_{\infty} \text { and see hypothesis } H(\beta)_{1}\right) \\
& \Rightarrow u_{\lambda} \geq 0, u_{\lambda} \neq 0 .
\end{aligned}
$$

Then equation (11) becomes

$$
\begin{aligned}
& \left\langle A\left(u_{\lambda}\right), h\right\rangle+\int_{\Omega} \xi(z) u_{\lambda}^{p-1} h d z+\int_{\partial \Omega} \beta(z) u_{\lambda}^{p-1} h d \sigma \\
& =\int_{\Omega}\left[\lambda u_{\lambda}^{p-1}+f\left(z, u_{\lambda}\right)\right] h d z \text { for all } h \in W^{1, p}(\Omega), \\
& \Rightarrow-\Delta_{p} u_{\lambda}(z)+\xi(z) u_{\lambda}(z)^{p-1}=\lambda u_{\lambda}(z)^{p-1}+f\left(z, u_{\lambda}(z)\right) \text { for a.a. } z \in \Omega, \\
& \quad \frac{\partial u_{\lambda}}{\partial n_{p}}+\beta(z) u_{\lambda}^{p-1}=0 \text { on } \partial \Omega, \\
& \Rightarrow u_{\lambda} \in S(\lambda) \subseteq D_{+} \quad \text { (see Proposition } 5 \text { and so } \mathcal{L}=\left(-\infty, \widehat{\lambda}_{1}\right) .
\end{aligned}
$$

In fact we can show that problem $\left(P_{\lambda}\right)$ for $\lambda<\widehat{\lambda}_{1}$ has a smallest positive solution.

Fix $\lambda<\widehat{\lambda}_{1}$ and $r \in\left(p, p^{*}\right)$. Hypotheses $H_{1}$ (i), (ii), (iii) imply that we can find $c_{7}(\lambda)>0$ with $\lambda \rightarrow c_{7}(\lambda)$ bounded on bounded subsets of $\mathcal{L}=\left(-\infty, \widehat{\lambda}_{1}\right)$ s.t.

$$
\lambda x^{p-1}+f(z, x) \geq c_{1} x^{q-1}-c_{7}(\lambda) x^{r-1} \text { for a.a. } z \in \Omega \text {, all } x \geq 0 .
$$

This unilateral growth estimate for the reaction term of problem $\left(P_{\lambda}\right)$ leads to the following auxiliary nonlinear Robin problem:

$$
\begin{cases}-\Delta_{p} u(z)+\xi(z) u(z)^{p-1}=c_{1} u(z)^{q-1}-c_{7}(\lambda) u(z)^{r-1} & \text { in } \Omega, \\ \frac{\partial u}{\partial n_{p}}+\beta(z) u^{p-1}=0 & \text { on } \partial \Omega, u \geq 0 .\end{cases}
$$

For this problem we have the following existence and uniqueness result.

Proposition 8 If hypotheses $H(\xi), H(\beta)_{1}$ hold, then for every $\lambda \in \mathbb{R}$ problem $\left(A u_{\lambda}\right)$ admits a unique positive solution $u_{*}^{\lambda} \in D_{+}$.

Proof First we show the existence of a positive solution for problem $\left(A u_{\lambda}\right)$. To this end, we consider the $C^{1}$-functional $\psi_{\lambda}: W^{1, p}(\Omega) \rightarrow \mathbb{R}$ defined by 


$$
\begin{aligned}
\psi_{\lambda}(u)= & \frac{1}{p} \vartheta(u)+\frac{\eta}{p}\left\|u^{-}\right\|_{p}^{p}-\frac{c_{1}}{q}\left\|u^{+}\right\|_{q}^{q}+\frac{c_{7}(\lambda)}{r}\left\|u^{+}\right\|_{r}^{r} \text { for all } u \in W^{1, p}(\Omega) \\
& \geq \frac{1}{p}\left[\vartheta\left(u^{-}\right)+\eta\left\|u^{-}\right\|_{p}^{p}\right]+\frac{1}{p} \vartheta\left(u^{+}\right)+\frac{c_{7}(\lambda)}{r}\left\|u^{+}\right\|_{r}^{r}-\frac{c_{1}}{q}\left\|u^{+}\right\|_{q}^{q} .
\end{aligned}
$$

We have

$$
\begin{aligned}
& \frac{1}{p} \vartheta\left(u^{+}\right)+\frac{c_{7}(\lambda)}{r}\left\|u^{+}\right\|_{r}^{r}-\frac{c_{1}}{q}\left\|u^{+}\right\|_{q}^{q} \\
& \left.\geq \frac{1}{p}\left\|\nabla u^{+}\right\|_{p}^{p}+c_{8}(\lambda)\left\|u^{+}\right\|_{p}^{r}-\frac{1}{p}\|\xi\|_{\infty}\left\|u^{+}\right\|_{p}^{p}-c_{9}\left\|u^{+}\right\|_{p}^{q} \quad \text { (for some } c_{8}(\lambda), c_{9}>0\right) \\
& \quad=\frac{1}{p}\left\|\nabla u^{+}\right\|_{p}^{p}+\left[c_{8}(\lambda)\left\|u^{+}\right\|_{p}^{r-p}-\frac{1}{p}\|\xi\|_{\infty}-\frac{c_{9}}{\left\|u^{+}\right\|_{p}^{p-q}}\right]\left\|u^{+}\right\|_{p}^{p} .
\end{aligned}
$$

Using (14) in (13) and recalling that $q<p<r$, we infer that $\psi_{\lambda}(\cdot)$ is coercive. Also, it is sequentially weakly lower semicontinuous (use the Sobolev embedding theorem and the compactness of the trace map). So, by the Weierstrass-Tonelli theorem, we can find $u_{*}^{\lambda} \in W^{1, p}(\Omega)$ s.t.

$$
\psi_{\lambda}\left(u_{*}^{\lambda}\right)=\inf \left[\psi_{\lambda}(u): u \in W^{1, p}(\Omega)\right]
$$

Since $q<p<r$, as before (see the proof of Proposition 7), we can show that

$$
\begin{gathered}
\psi_{\lambda}\left(u_{*}^{\lambda}\right)<0, \\
\Rightarrow u_{*}^{\lambda} \neq 0 .
\end{gathered}
$$

From (15) we have

$$
\begin{aligned}
\psi_{\lambda}^{\prime}\left(u_{*}^{\lambda}\right)=0 & \\
\Rightarrow & \left\langle A\left(u_{*}^{\lambda}\right), h\right\rangle+\int_{\Omega} \xi(z)\left|u_{*}^{\lambda}\right|^{p-2} u_{*}^{\lambda} h d z+\int_{\partial \Omega} \beta(z)\left|u_{*}^{\lambda}\right|^{p-2} u_{*}^{\lambda} h d \sigma \\
& -\eta \int_{\Omega}\left(u_{*}^{\lambda^{-}}\right)^{p-1} h d z \\
= & c_{1} \int_{\Omega}\left(u_{*}^{\lambda^{+}}\right)^{q-1} h d z-c_{7}(\lambda) \int_{\Omega}\left(u_{*}^{\lambda^{+}}\right)^{r-1} h d z \text { for all } h \in W^{1, p}(\Omega) .
\end{aligned}
$$

In (16) we choose $h=-u_{*}^{\lambda^{-}} \in W^{1, p}(\Omega)$. Then

$$
\begin{aligned}
& \vartheta\left(u_{*}^{\lambda^{-}}\right)+\eta\left\|u_{*}^{\lambda^{-}}\right\|_{p}^{p}=0 \\
& \left.\quad \Rightarrow c_{10}\left\|u_{*}^{\lambda^{-}}\right\|^{p} \leq 0 \text { for some } c_{10}>0 \text { (recall that } \eta>\|\xi\|_{\infty}\right) \\
& \quad \Rightarrow u_{*}^{\lambda} \geq 0, u_{*}^{\lambda} \neq 0 .
\end{aligned}
$$


Therefore Eq. (16) becomes

$$
\begin{aligned}
\left\langle A\left(u_{*}^{\lambda}\right), h\right\rangle+\int_{\Omega} \xi(z)\left(u_{*}^{\lambda}\right)^{p-1} h d z+\int_{\partial \Omega} \beta(z)\left(u_{*}^{\lambda}\right)^{p-1} h d \sigma \\
=c_{1} \int_{\Omega}\left(u_{*}^{\lambda}\right)^{q-1} h d z-c_{7}(\lambda) \int_{\Omega}\left(u_{*}^{\lambda}\right)^{r-1} h d z \quad \text { for all } h \in W^{1, p}(\Omega), \\
\Rightarrow-\Delta_{p} u_{*}^{\lambda}(z)+\xi(z) u_{*}^{\lambda}(z)^{p-1}=c_{1} u_{*}^{\lambda}(z)^{q-1}-c_{7}(\lambda) u_{*}^{\lambda}(z)^{r-1} \quad \text { for a.a. } z \in \Omega, \\
\\
\quad \frac{\partial u_{*}^{\lambda}}{\partial n_{p}}+\beta(z)\left(u_{*}^{\lambda}\right)^{p-1}=0 \text { on } \partial \Omega \quad(\text { see Papageorgiou-Rădulescu [17]), }, \text { (17) } \\
\Rightarrow u_{*}^{\lambda} \text { is a positive solution of }\left(A u_{\lambda}\right) .
\end{aligned}
$$

As before, the nonlinear regularity theory (see [13]) implies $u_{*}^{\lambda} \in C_{+} \backslash\{0\}$.

Moreover, from (17) we have

$$
\begin{aligned}
\Delta_{p} u_{*}^{\lambda}(z) & \leq\left(c_{7}(\lambda)\left\|u_{*}^{\lambda}\right\|_{\infty}^{r-p}+\|\xi\|_{\infty}\right) u_{*}^{\lambda}(z)^{p-1} \\
& \leq c_{11} u_{*}^{\lambda}(z)^{p-1} \text { for a.a. } z \in \Omega, \text { some } c_{11}>0, \\
& \Rightarrow u_{*}^{\lambda} \in D_{+}(\text {by the nonlinear strong maximum principle, [8] (p. 738)). }
\end{aligned}
$$

Next we show the uniqueness of this positive solution. To this end suppose that $v_{*}^{\lambda} \in W^{1, p}(\Omega)$ is another positive solution of $\left(A u_{\lambda}\right)$. As above we can show that $v_{*}^{\lambda} \in D_{+}$.

We have

$$
\begin{aligned}
\int_{\Omega} & \left(\frac{c_{1}}{\left(u_{*}^{\lambda}\right)^{p-q}}-c_{7}(\lambda)\left(u_{*}^{\lambda}\right)^{r-p}\right)\left(\left(u_{*}^{\lambda}\right)^{p}-\left(v_{*}^{\lambda}\right)^{p}\right) d z \\
= & \int_{\Omega}\left(c_{1}\left(u_{*}^{\lambda}\right)^{q-1}-c_{7}(\lambda)\left(u_{*}^{\lambda}\right)^{r-1}\right)\left(u_{*}^{\lambda}-\frac{\left(v_{*}^{\lambda}\right)^{p}}{\left(u_{*}^{\lambda}\right)^{p-1}}\right) d z \\
= & \int_{\Omega}\left(-\Delta_{p} u_{*}^{\lambda}+\xi(z)\left(u_{*}^{\lambda}\right)^{p-1}\right)\left(u_{*}^{\lambda}-\frac{\left(v_{*}^{\lambda}\right)^{p}}{\left(u_{*}^{\lambda}\right)^{p-1}}\right) d z \quad(\text { see }(17)) \\
= & \int_{\Omega}\left|\nabla u_{*}^{\lambda}\right|^{p-2}\left(\nabla u_{*}^{\lambda}, \nabla\left(u_{*}^{\lambda}-\frac{\left(v_{*}^{\lambda}\right)^{p}}{\left(u_{*}^{\lambda}\right)^{p-1}}\right)\right)_{\mathbb{R}^{N}} d z \\
& +\int_{\Omega} \xi(z)\left(u_{*}^{\lambda}\right)^{p-1}\left(u_{*}^{\lambda}-\frac{\left(v_{*}^{\lambda}\right)^{p}}{\left(u_{*}^{\lambda}\right)^{p-1}}\right) d z \\
& +\int_{\Omega} \beta(z)\left(u_{*}^{\lambda}\right)^{p-1}\left(u_{*}^{\lambda}-\frac{\left(v_{*}^{\lambda}\right)^{p}}{\left(u_{*}^{\lambda}\right)^{p-1}}\right) d \sigma \\
& (\mathrm{using} \text { the nonlinear Green's identity, see }[8](\mathrm{p} .211)) \\
= & \left\|\nabla u_{*}^{\lambda}\right\|_{p}^{p}-\left\|\nabla v_{*}^{\lambda}\right\|_{p}^{p}+\int_{\Omega} R\left(v_{*}^{\lambda}, u_{*}^{\lambda}\right) d z+\int_{\Omega} \xi(z)\left(\left(u_{*}^{\lambda}\right)^{p}-\left(v_{*}^{\lambda}\right)^{p}\right) d z \\
& +\int_{\Omega} \beta(z)\left(\left(u_{*}^{\lambda}\right)^{p}-\left(v_{*}^{\lambda}\right)^{p}\right) d \sigma .
\end{aligned}
$$


Interchanging the roles of $u_{*}^{\lambda}$ and $v_{*}^{\lambda}$ in the above argument, we also have

$$
\begin{aligned}
\int_{\Omega} & \left(\frac{c_{1}}{\left(v_{*}^{\lambda}\right)^{p-q}}-c_{7}(\lambda)\left(v_{*}^{\lambda}\right)^{r-p}\right)\left(\left(v_{*}^{\lambda}\right)^{p}-\left(u_{*}^{\lambda}\right)^{p}\right) d z \\
= & \left\|\nabla v_{*}^{\lambda}\right\|_{p}^{p}-\left\|\nabla u_{*}^{\lambda}\right\|_{p}^{p}+\int_{\Omega} R\left(u_{*}^{\lambda}, v_{*}^{\lambda}\right) d z+\int_{\Omega} \xi(z)\left(\left(v_{*}^{\lambda}\right)^{p}-\left(u_{*}^{\lambda}\right)^{p}\right) d z \\
& +\int_{\Omega} \beta(z)\left(\left(v_{*}^{\lambda}\right)^{p}-\left(u_{*}^{\lambda}\right)^{p}\right) d \sigma .
\end{aligned}
$$

Adding (18) and (19) and using the nonlinear Picone's identity, we have

$$
\begin{aligned}
0 & \leq \int_{\Omega}\left(R\left(v_{*}^{\lambda}, u_{*}^{\lambda}\right)+R\left(u_{*}^{\lambda}, v_{*}^{\lambda}\right)\right) d z \\
& =\int_{\Omega}\left(c_{1}\left(\frac{1}{\left(u_{*}^{\lambda}\right)^{p-q}}-\frac{1}{\left(v_{*}^{\lambda}\right)^{p-q}}\right)-c_{7}(\lambda)\left(\left(u_{*}^{\lambda}\right)^{r-p}-\left(v_{*}^{\lambda}\right)^{r-p}\right)\right)\left(\left(u_{*}^{\lambda}\right)^{p}-\left(v_{*}^{\lambda}\right)^{p}\right) d z .
\end{aligned}
$$

Since the function $x \rightarrow \frac{c_{1}}{x^{p-q}}-c_{7}(\lambda) x^{r-p}$ is strictly decreasing on $(0,+\infty)$, from (20) we infer that

$$
u_{*}^{\lambda}=v_{*}^{\lambda} .
$$

This proves the uniqueness of the positive solution $u_{*}^{\lambda} \in D_{+}$of problem $\left(A u_{\lambda}\right)$.

Remark 3 There is an alternative approach to the uniqueness of the positive solution $u_{*}^{\lambda} \in D_{+}$of problem $\left(A u_{\lambda}\right)$ which does not use the nonlinear Picone's identity. For this we need to assume that $\beta(z)>0$ for all $z \in \partial \Omega$. First note that, if $\rho=\left\|u_{*}^{\lambda}\right\|_{\infty}$, then we can find $\widehat{\xi}_{\rho}>0$ s.t. for a.a. $z \in \Omega$, the function $x \rightarrow c_{1} x^{q-1}-c_{7}(\lambda) x^{r-1}+\widehat{\xi}_{\rho} x^{p-1}$ is nondecreasing on $[0, \rho]$. As before let $v_{*}^{\lambda} \in D_{+}$be another positive solution of $\left(A u_{\lambda}\right)$ and let $t>0$ be the biggest real s.t.

$$
t v_{*}^{\lambda} \leq u_{*}^{\lambda} .
$$

We assume that $t \in(0,1)$. We have

$$
\begin{aligned}
- & \Delta_{p}\left(t v_{*}^{\lambda}\right)+\left(\xi(z)+\widehat{\xi}_{\rho}\right)\left(t v_{*}^{\lambda}\right)^{p-1} \\
& =t^{p-1}\left[-\Delta_{p} v_{*}^{\lambda}+\left(\xi(z)+\widehat{\xi}_{\rho}\right)\left(v_{*}^{\lambda}\right)^{p-1}\right] \\
& =t^{p-1}\left[c_{1}\left(v_{*}^{\lambda}\right)^{q-1}-c_{7}(\lambda)\left(v_{*}^{\lambda}\right)^{r-1}+\widehat{\xi}_{\rho}\left(v_{*}^{\lambda}\right)^{p-1}\right] \\
& <c_{1}\left(t v_{*}^{\lambda}\right)^{q-1}-c_{7}(\lambda)\left(t v_{*}^{\lambda}\right)^{r-1}+\widehat{\xi}_{\rho}\left(t v_{*}^{\lambda}\right)^{p-1} \quad(\text { since } t \in(0,1) \text { and } q<p<r) \\
& \leq c_{1}\left(u_{*}^{\lambda}\right)^{q-1}-c_{7}(\lambda)\left(u_{*}^{\lambda}\right)^{r-1}+\widehat{\xi}_{\rho}\left(u_{*}^{\lambda}\right)^{p-1} \quad(\text { see }(21)) \\
& =-\Delta_{p} u_{*}^{\lambda}+\left(\xi(z)+\widehat{\xi}_{\rho}\right)\left(u_{*}^{\lambda}\right)^{p-1} \quad \text { for a.a. } z \in \Omega .
\end{aligned}
$$


Invoking Proposition 4 (recall $\beta>0$ ), we have

$$
u_{*}^{\lambda}-t v_{*}^{\lambda} \in D_{+},
$$

where we recall that $\widehat{C}_{+}=\left\{y \in C^{1}(\bar{\Omega}): y(z) \geq 0\right.$ for all $\left.z \in \bar{\Omega},\left.\frac{\partial u}{\partial n}\right|_{\partial \Omega} \leq 0\right\}$.

Evidently (22) contradicts the maximality of $t>0$. Therefore we must have $t \geq 1$ and so

$$
v_{*}^{\lambda} \leq u_{*}^{\lambda}(\operatorname{see}(21))
$$

Interchanging the roles of $u_{*}^{\lambda} \in D_{+}$and $v_{*}^{\lambda} \in D_{+}$in the above argument we also have

$$
\begin{aligned}
& u_{*}^{\lambda} \leq v_{*}^{\lambda}, \\
& \quad \Rightarrow u_{*}^{\lambda}=v_{*}^{\lambda} .
\end{aligned}
$$

So, again we have proved uniqueness of the positive solution of problem $\left(A u_{\lambda}\right)$. Recall that $\lambda \rightarrow c_{7}(\lambda)$ is bounded on bounded sets of $\lambda \in \mathbb{R}$. So, if $B \subseteq \mathbb{R}$ is bounded, $\widehat{c}_{7} \geq c_{7}(\lambda)$ for all $\lambda \in B$ and $\widehat{u} \in D_{+}$is the unique positive solution of the auxiliary problem

$$
\begin{cases}-\Delta_{p} u(z)+\xi(z) u(z)^{p-1}=c_{1} u(z)^{q-1}-\widehat{c}_{7} u(z)^{r-1} & \text { in } \Omega, \\ \frac{\partial u}{\partial n_{p}}+\beta(z) u^{p-1}=0 & \text { on } \partial \Omega\end{cases}
$$

(see Proposition 8), then $\widehat{u} \leq u_{*}^{\lambda}$ for all $\lambda \in B$.

Next using $u_{*}^{\lambda} \in D_{+}$, we can have a lower bound for the elements of the set $S(\lambda)$. This fact will be used to produce the smallest positive solution for problem $\left(P_{\lambda}\right)$ when $\lambda<\widehat{\lambda}_{1}$.

So, we have the following result.

Proposition 9 If hypotheses $H(\xi), H(\beta)_{1}, H_{1}$ hold and $\lambda<\widehat{\lambda}_{1}$, then $u_{*}^{\lambda} \leq u$ for all $u \in S(\lambda)$.

Proof As before let $\eta>\|\xi\|_{\infty}$. For $u \in S(\lambda)$ we consider the following Carathéodory function

$$
\widehat{g}_{\lambda}(z, x)= \begin{cases}0 & \text { if } x<0 \\ c_{1} x^{q-1}-c_{7}(\lambda) x^{r-1}+\eta x^{p-1} & \text { if } 0 \leq x \leq u(z) \\ c_{1} u(z)^{q-1}-c_{7}(\lambda) u(z)^{r-1}+\eta u(z)^{p-1} & \text { if } u(z)<x\end{cases}
$$

We set $\widehat{G}_{\lambda}(z, x)=\int_{0}^{x} \widehat{g}_{\lambda}(z, s) d s$ and consider the $C^{1}$-functional $\widehat{\psi}_{\lambda}: W^{1, p}(\Omega) \rightarrow$ $\mathbb{R}$ defined by

$$
\widehat{\psi}_{\lambda}(u)=\frac{1}{p} \vartheta(u)+\frac{\eta}{p}\|u\|_{p}^{p}-\int_{\Omega} \widehat{G}_{\lambda}(z, u) d z \text { for all } u \in W^{1, p}(\Omega) .
$$


From (23) and since $\eta>\|\xi\|_{\infty}$, we see that the functional $\widehat{\psi}_{\lambda}$ is coercive. Also, it is sequentially weakly lower semicontinuous. So, we can find $\widehat{u}_{*}^{\lambda} \in W^{1, p}(\Omega)$ s.t.

$$
\widehat{\psi}_{\lambda}\left(\widehat{u}_{*}^{\lambda}\right)=\inf \left[\widehat{\psi}_{\lambda}(u): u \in W^{1, p}(\Omega)\right] .
$$

As before, since $q<p<r$, we have that

$$
\begin{aligned}
& \widehat{\psi}_{\lambda}\left(\widehat{u}_{*}^{\lambda}\right)<0=\widehat{\psi}_{\lambda}(0), \\
& \Rightarrow \widehat{u}_{*}^{\lambda} \neq 0 .
\end{aligned}
$$

From (24) we have

$$
\begin{aligned}
& \widehat{\psi}_{\lambda}^{\prime}\left(\widehat{u}_{*}^{\lambda}\right)=0, \\
& \quad \Rightarrow\left\langle A\left(\widehat{u}_{*}^{\lambda}\right), h\right\rangle+\int_{\Omega}(\xi(z)+\eta)\left|\widehat{u}_{*}^{\lambda}\right|^{p-2} \widehat{u}_{*}^{\lambda} h d z+\int_{\partial \Omega} \beta(z)\left|\widehat{u}_{*}^{\lambda}\right|^{p-2} \widehat{u}_{*}^{\lambda} h d \sigma \\
& \quad=\int_{\Omega} g_{\lambda}\left(z, \widehat{u}_{*}^{\lambda}\right) h d z \text { for all } h \in W^{1, p}(\Omega) .
\end{aligned}
$$

In (25) first we choose $h=-\widehat{u}_{*}^{\lambda^{-}} \in W^{1, p}(\Omega)$. We obtain

$$
\begin{aligned}
& \vartheta\left(\widehat{u}_{*}^{\lambda^{-}}\right)+\eta\left\|\widehat{u}_{*}^{\lambda^{-}}\right\|_{p}^{p}=0 \quad(\text { see }(23)), \\
& \quad \Rightarrow c_{12}\left\|\widehat{u}_{*}^{\lambda^{-}}\right\|^{p} \leq 0 \quad \text { for some } c_{12}>0\left(\text { recall that } \eta>\|\xi\|_{\infty}\right), \\
& \quad \Rightarrow \widehat{u}_{*}^{\lambda} \geq 0, \widehat{u}_{*}^{\lambda} \neq 0 .
\end{aligned}
$$

Next in (25) we choose $\left(\widehat{u}_{*}^{\lambda}-u\right)^{+} \in W^{1, p}(\Omega)$. We have

$$
\begin{aligned}
\left\langle A\left(\widehat{u}_{*}^{\lambda}\right),\left(\widehat{u}_{*}^{\lambda}-u\right)^{+}\right\rangle+\int_{\Omega}(\xi(z)+\eta)\left(\widehat{u}_{*}^{\lambda}\right)^{p-1}\left(\widehat{u}_{*}^{\lambda}-u\right)^{+} d z \\
\quad+\int_{\partial \Omega} \beta(z)\left(\widehat{u}_{*}^{\lambda}\right)^{p-1}\left(\widehat{u}_{*}^{\lambda}-u\right)^{+} d \sigma \\
=\int_{\Omega}\left(c_{1} u^{q-1}-c_{7}(\lambda) u^{r-1}+\eta u^{p-1}\right)\left(\widehat{u}_{*}^{\lambda}-u\right)^{+} d z \quad(\text { see }(23)) \\
\leq \int_{\Omega}\left(\lambda u^{p-1}+f(z, u)+\eta u^{p-1}\right)\left(\widehat{u}_{*}^{\lambda}-u\right)^{+} d z \quad(\text { see }(12)) \\
=\left\langle A(u),\left(\widehat{u}_{*}^{\lambda}-u\right)^{+}\right\rangle+\int_{\Omega}(\xi(z)+\eta) u^{p-1}\left(\widehat{u}_{*}^{\lambda}-u\right)^{+} d z \\
\quad+\int_{\partial \Omega} \beta(z) u^{p-1}\left(\widehat{u}_{*}^{\lambda}-u\right)^{+} d \sigma(\operatorname{recall~that~} u \in S(\lambda)), \\
\Rightarrow\left\langle A\left(\widehat{u}_{*}^{\lambda}\right)-A(u),\left(\widehat{u}_{*}^{\lambda}-u\right)^{+}\right\rangle+\int_{\Omega}(\xi(z)+\eta)\left(\left(\widehat{u}_{*}^{\lambda}\right)^{p-1}-u^{p-1}\right)\left(\widehat{u}_{*}^{\lambda}-u\right)^{+} d z \\
\quad+\int_{\partial \Omega} \beta(z)\left(\left(\widehat{u}_{*}^{\lambda}\right)^{p-1}-u^{p-1}\right)\left(\widehat{u}_{*}^{\lambda}-u\right)^{+} d \sigma \leq 0,
\end{aligned}
$$


$\Rightarrow \widehat{u}_{*}^{\lambda} \leq u \quad\left(\right.$ since $\eta>\|\xi\|_{\infty}$ and $\beta \geq 0$, see hypothesis $\left.H(\beta)_{1}\right)$.

Therefore, we have proved that

$$
\begin{aligned}
\widehat{u}_{*}^{\lambda} & \in[0, u]=\left\{v \in W^{1, p}(\Omega): 0 \leq v(z) \leq u(z) \text { for a.a. } z \in \Omega\right\}, \widehat{u}_{*}^{\lambda} \neq 0, \\
& \Rightarrow \widehat{u}_{*}^{\lambda} \text { is a positive solution of }\left(A u_{\lambda}\right)(\text { see }(25) \text { and }(23)), \\
& \Rightarrow \widehat{u}_{*}^{\lambda}=u_{*}^{\lambda} \in D_{+} \quad(\text { see Proposition } 8) .
\end{aligned}
$$

Finally we have

$$
u_{*}^{\lambda} \leq u \text { for all } u \in S(\lambda)
$$

Proposition 10 If hypotheses $H(\xi), H(\beta)_{1}, H_{1}$ hold and $\lambda<\widehat{\lambda}_{1}$, then problem $\left(P_{\lambda}\right)$ admits a smallest positive solution $\bar{u}_{\lambda} \in D_{+}$.

Proof As in Filippakis-Papageorgiou [5], we have that $S(\lambda)$ is downward directed, that is, if $u_{1}, u_{2} \in S(\lambda)$, there is $u \in S(\lambda)$ s.t. $u \leq u_{1}, u \leq u_{2}$. Invoking Lemma 3.10 of Hu-Papageorgiou [9] (p. 178), we can find $\left\{u_{n}\right\}_{n \geq 1} \subseteq S(\lambda)$ decreasing s.t.

$$
\inf S(\lambda)=\inf _{n \geq 1} u_{n}
$$

We have

$$
\left\langle A\left(u_{n}\right), h\right\rangle+\int_{\Omega} \xi(z) u_{n}^{p-1} h d z+\int_{\partial \Omega} \beta(z) u_{n}^{p-1} h d \sigma=\int_{\Omega}\left(\lambda u_{n}^{p-1}+f\left(z, u_{n}\right)\right) d z
$$

for all $h \in W^{1, p}(\Omega)$. Since $u_{n} \leq u_{1} \in S(\lambda) \subseteq D_{+}$, from (26) and hypotheses $H(\xi)$, $H(\beta)_{1}, H_{1}(\mathrm{i})$ it follows that

$$
\left\{u_{n}\right\}_{n \geq 1} \subseteq W^{1, p}(\Omega) \text { is bounded. }
$$

So, we may assume that

$$
u_{n} \stackrel{w}{\rightarrow} \bar{u}_{\lambda} \text { in } W^{1, p}(\Omega) \text { and } u_{n} \rightarrow \bar{u}_{\lambda} \text { in } L^{p}(\Omega) \text { and in } L^{p}(\partial \Omega) .
$$

In (26) we choose $h=u_{n}-\bar{u}_{\lambda} \in W^{1, p}(\Omega)$, pass to the limit as $n \rightarrow+\infty$ and use (27). Then we have

$$
\begin{aligned}
& \lim _{n \rightarrow+\infty}\left\langle A\left(u_{n}\right), u_{n}-\bar{u}_{\lambda}\right\rangle=0, \\
& \quad \Rightarrow u_{n} \rightarrow \bar{u}_{\lambda} \text { in } W^{1, p}(\Omega) \text { (see Proposition 2). }
\end{aligned}
$$


If in (26) we pass to the limit as $n \rightarrow+\infty$ and use (28), then

$$
\begin{aligned}
& \left\langle A\left(\bar{u}_{\lambda}\right), h\right\rangle+\int_{\Omega} \xi(z) \bar{u}_{\lambda}^{p-1} h d z+\int_{\partial \Omega} \beta(z) \bar{u}_{\lambda}^{p-1} h d \sigma=\int_{\Omega}\left(\lambda \bar{u}_{\lambda}^{p-1}+f\left(z, \bar{u}_{\lambda}\right)\right) h d z \\
& \text { for all } h \in W^{1, p}(\Omega), \\
& \quad \Rightarrow \bar{u}_{\lambda} \geq 0 \text { is a solution of problem }\left(P_{\lambda}\right) .
\end{aligned}
$$

From Proposition 9 we have

$$
\begin{aligned}
& u_{*}^{\lambda} \leq u_{n} \text { for all } n \in \mathbb{N} \\
& \quad \Rightarrow u_{*}^{\lambda} \leq \bar{u}_{\lambda} \quad(\operatorname{see}(28))
\end{aligned}
$$

Hence $\bar{u}_{\lambda} \neq 0$ and so we conclude that

$$
\bar{u}_{\lambda} \in S(\lambda) \subseteq D_{+} \text {and } \bar{u}_{\lambda}=\inf S(\lambda) .
$$

Next we examine the map $\lambda \rightarrow \bar{u}_{\lambda}$ from $\left(-\infty, \widehat{\lambda}_{1}\right)$ into $C^{1}(\bar{\Omega})$.

Proposition 11 If hypotheses $H(\xi), H(\beta)_{1}, H_{1}$ hold, then the map $\lambda \rightarrow \bar{u}_{\lambda}$ from $\mathcal{L}=\left(-\infty, \widehat{\lambda}_{1}\right)$ into $C^{1}(\bar{\Omega})$ is nondecreasing (that is, if $\lambda<\mu$, then $\bar{u}_{\lambda} \leq \bar{u}_{\mu}$ ) and left continuous.

Proof Suppose that $\lambda, \mu \in \mathcal{L}=\left(-\infty, \widehat{\lambda}_{1}\right)$ and $\lambda<\mu$. Let $\bar{u}_{\mu} \in S(\mu)$ be the minimal positive solution of problem $\left(P_{\mu}\right)$ (see Proposition 10). For $\eta>\|\xi\|_{\infty}$ we introduce the following Carathéodory function

$$
e_{\lambda}(z, x)= \begin{cases}0 & \text { if } x<0 \\ (\lambda+\eta) x^{p-1}+f(z, x) & \text { if } 0 \leq x \leq \bar{u}_{\mu}(z), \\ (\lambda+\eta) \bar{u}_{\mu}(z)^{p-1}+f\left(z, \bar{u}_{\mu}(z)\right) & \text { if } \bar{u}_{\mu}(z)<x\end{cases}
$$

We set $E_{\lambda}(z, x)=\int_{0}^{x} e_{\lambda}(z, s) d s$ and consider the $C^{1}$-functional $\widetilde{\psi}_{\lambda}: W^{1, p}(\Omega) \rightarrow$ $\mathbb{R}$ defined by

$$
\widetilde{\psi}_{\lambda}(u)=\frac{1}{p} \vartheta(u)+\frac{\eta}{p}\|u\|_{p}^{p}-\int_{\Omega} E_{\lambda}(z, u) d z \text { for all } u \in W^{1, p}(\Omega) .
$$

From (29) and since $\eta>\|\xi\|_{\infty}$, we see that $\widetilde{\psi}_{\lambda}$ is coercive. Also, it is sequentially weakly lower semicontinuous. So, we can find $u_{\lambda} \in W^{1, p}(\Omega)$ s.t.

$$
\widetilde{\psi}_{\lambda}\left(u_{\lambda}\right)=\inf \left[\widetilde{\psi}_{\lambda}(u): u \in W^{1, p}(\Omega)\right] .
$$

Let $m_{\mu}=\min _{\bar{\Omega}} \bar{u}_{\mu}>0$ (recall that $\left.\bar{u}_{\mu} \in D_{+}\right)$and choose $t \in(0,1)$ small s.t. $t \widehat{u}_{1}(z) \leq \min \left\{m_{\mu}, \delta\right\}$ for all $z \in \bar{\Omega}$ (here $\delta>0$ is as in hypothesis $H_{1}$ (iii)). Because $q<p$ and by choosing $t \in(0,1)$ even smaller if necessary, we have that

$$
\widetilde{\psi}_{\lambda}\left(t \widehat{u}_{1}\right)<0
$$




$$
\begin{aligned}
& \Rightarrow \widetilde{\psi}_{\lambda}\left(u_{\lambda}\right)<0=\widetilde{\psi}_{\lambda}(0) \quad(\text { see }(30)) \\
& \Rightarrow u_{\lambda} \neq 0 .
\end{aligned}
$$

From (30) we have

$$
\begin{aligned}
& \widetilde{\psi}_{\lambda}^{\prime}\left(u_{\lambda}\right)=0, \\
& \quad \Rightarrow\left\langle A\left(u_{\lambda}\right), h\right\rangle+\int_{\Omega}(\xi(z)+\eta)\left|u_{\lambda}\right|^{p-2} u_{\lambda} h d z+\int_{\partial \Omega} \beta(z)\left|u_{\lambda}\right|^{p-2} u_{\lambda} h d \sigma \\
& \quad=\int_{\Omega} e_{\lambda}\left(z, u_{\lambda}\right) h d z \text { for all } h \in W^{1, p}(\Omega) .
\end{aligned}
$$

As in the proof of Proposition 9, using this time (31) and (29), we show that

$$
\begin{aligned}
& u_{\lambda} \in\left[0, \bar{u}_{\mu}\right]=\left\{v \in W^{1, p}(\Omega): 0 \leq v(z) \leq \bar{u}_{\mu}(z) \text { for a.a. } z \in \Omega\right\}, \quad u_{\lambda} \neq 0, \\
& \quad \Rightarrow u_{\lambda} \in S(\lambda) \subseteq D_{+} \quad(\operatorname{see}(29),(31)), \\
& \quad \Rightarrow \bar{u}_{\lambda} \leq \bar{u}_{\mu}
\end{aligned}
$$

This proves that $\lambda \rightarrow \bar{u}_{\lambda}$ is nondecreasing.

Next we show the left continuity of this map. So, let $\left\{\lambda_{n}, \lambda\right\}_{n \geq 1} \subseteq \mathcal{L}$ and suppose that $\lambda_{n} \rightarrow \lambda^{-}$. From the first part of the proof we have $\bar{u}_{\lambda_{n}} \leq \bar{u}_{\lambda}$ for all $n \in \mathbb{N}$ and so we infer that $\left\{\bar{u}_{\lambda_{n}}\right\}_{n \geq 1} \subseteq W^{1, p}(\Omega)$ is bounded. So, we may assume that

$$
\bar{u}_{\lambda_{n}} \stackrel{w}{\rightarrow} \widetilde{u} \text { in } W^{1, p}(\Omega) \text { and } \bar{u}_{\lambda_{n}} \rightarrow \widetilde{u} \text { in } L^{p}(\Omega) \text { and in } L^{p}(\partial \Omega)
$$

We have

$$
\left\langle A\left(\bar{u}_{\lambda_{n}}\right), h\right\rangle+\int_{\Omega} \xi(z) \bar{u}_{\lambda_{n}}^{p-1} h d z+\int_{\partial \Omega} \beta(z) \bar{u}_{\lambda_{n}}^{p-1} h d \sigma=\int_{\Omega}\left(\lambda_{n} \bar{u}_{\lambda_{n}}^{p-1}+f\left(z, \bar{u}_{\lambda_{n}}\right)\right) h d z
$$

for all $h \in W^{1, p}(\Omega)$, all $n \in \mathbb{N}$. In (33) we choose $h=\bar{u}_{\lambda_{n}}-\tilde{u} \in W^{1, p}(\Omega)$, pass to the limit as $n \rightarrow+\infty$ and use (32). Then

$$
\begin{aligned}
& \lim _{n \rightarrow+\infty}\left\langle A\left(\bar{u}_{\lambda_{n}}\right), \bar{u}_{\lambda_{n}}-\widetilde{u}\right\rangle=0 \\
& \quad \Rightarrow \bar{u}_{\lambda_{n}} \rightarrow \tilde{u} \text { in } W^{1, p}(\Omega) \quad(\text { see Proposition } 2)
\end{aligned}
$$

So, if in (33) we pass to the limit as $n \rightarrow+\infty$ and use (34), then 


$$
\langle A(\tilde{u}), h\rangle+\int_{\Omega} \xi(z) \tilde{u}^{p-1} h d z+\int_{\partial \Omega} \beta(z) \tilde{u}^{p-1} h d \sigma=\int_{\Omega}\left(\lambda \widetilde{u}^{p-1}+f(z, \tilde{u})\right) h d z
$$

for all $h \in W^{1, p}(\Omega)$.

Set $B=\left\{\lambda_{n}\right\}_{n \geq 1}$ and let $\widehat{c}_{7} \geq c_{7}(\tilde{\lambda})$ for all $\tilde{\lambda} \in B$ (recall that $\lambda \rightarrow c_{7}(\lambda)$ is bounded on bounded sets). Consider $\widehat{u} \in D_{+}$the unique positive solution of

$$
\begin{cases}-\Delta_{p} u(z)+\xi(z) u(z)^{p-1}=c_{1} u(z)^{q-1}-\widehat{c}_{7} u(z)^{r-1} & \text { in } \Omega \\ \frac{\partial u}{\partial n_{p}}+\beta(z) u^{p-1}=0 & \text { on } \partial \Omega\end{cases}
$$

(see Proposition 8 and the Remark following it).

We know that

$$
\begin{aligned}
& \widehat{u} \leq \bar{u}_{\lambda_{n}} \text { for all } n \in \mathbb{N}, \\
& \quad \Rightarrow \widehat{u} \leq \tilde{u}, \text { that is, } \tilde{u} \neq 0 .
\end{aligned}
$$

Then from (35) we infer that $\tilde{u} \in S(\lambda)$.

Suppose that $\tilde{u} \neq \bar{u}_{\lambda}$. Then we can find $z_{0} \in \bar{\Omega}$ s.t.

$$
\bar{u}_{\lambda}\left(z_{0}\right)<\tilde{u}\left(z_{0}\right)
$$

From Theorem 2 of Lieberman [13], we know that there exist $M>0$ and $\tau \in(0,1)$ s.t.

$$
\bar{u}_{\lambda_{n}} \in C^{1, \tau}(\bar{\Omega}) \text { and }\left\|\bar{u}_{\lambda_{n}}\right\|_{C^{1, \tau}(\bar{\Omega})} \leq M \text { for all } n \in \mathbb{N}
$$

Exploiting the compact embedding of $C^{1, \tau}(\bar{\Omega})$ into $C^{1}(\bar{\Omega})$ and using (34), from (37) we have

$$
\begin{aligned}
& \bar{u}_{\lambda_{n}} \rightarrow \widetilde{u} \quad \text { in } C^{1}(\bar{\Omega}) \\
& \quad \Rightarrow \bar{u}_{\lambda_{n}}\left(z_{0}\right)>\bar{u}_{\lambda}\left(z_{0}\right), \quad \text { for all } n \geq n_{0} \quad(\text { see }(36))
\end{aligned}
$$

which contradicts the monotonicity of $\lambda \rightarrow \bar{u}_{\lambda}$ (recall $\lambda_{n}<\lambda$ for all $n \in \mathbb{N}$ ). Therefore $\tilde{u}=\bar{u}_{\lambda}$ and so from (38) we conclude that the map $\lambda \rightarrow \bar{u}_{\lambda}$ is left continuous from $\mathcal{L}=\left(-\infty, \widehat{\lambda}_{1}\right)$ into $C^{1}(\bar{\Omega})$.

If we strengthen the conditions on the perturbation $f(z, \cdot)$, we can have uniqueness of the positive solution for problem $\left(P_{\lambda}\right), \lambda<\widehat{\lambda}_{1}$.

The new hypotheses on $f(z, x)$ are the following:

$H_{2}: f: \Omega \times \mathbb{R} \rightarrow \mathbb{R}$ is a Carathéodory function s.t. for a.a. $z \in \Omega, f(z, 0)=0$, $f(z, x)>0$ for all $x>0$, hypotheses $H_{2}$ (i), (ii), (iii) are the same as the corresponding hypotheses $H_{1}(\mathrm{i})$, (ii), (iii) and

(iv) for a.a. $z \in \Omega$ the function $x \rightarrow \frac{f(z, x)}{x^{p-1}}$ is strictly decreasing on $(0,+\infty)$. 
Example 2 The function $f_{1}(x)=x^{q-1}$ for all $x \geq 0$ with $1<q<p$ satisfies hypotheses $H_{2}$. On the other hand the function

$$
f_{2}(x)=\left\{\begin{array}{ll}
x^{q-1}-x^{\tau-1} & \text { if } x \in[0,1], \\
\ln x^{p-1} & \text { if } 1<x,
\end{array} \text { with } 1<q<p, q<\tau,\right.
$$

need not satisfy hypotheses $H_{2}$ unless additional restrictions are imposed on the exponents $q, \tau$.

Proposition 12 If hypotheses $H(\xi), H(\beta)_{1}, H_{2}$ hold and $\lambda<\widehat{\lambda}_{1}$, then problem $\left(P_{\lambda}\right)$ has a unique positive solution $u_{\lambda} \in D_{+}$.

Proof Existence follows from Proposition 7. The uniqueness is proved as in the proof of Proposition 8 using the nonlinear Picone's identity (for an alternative approach, see the Remark following the proof of Proposition 8).

In this case, because of the uniqueness of the positive solution, Proposition 11 takes the following form:

Proposition 13 If hypotheses $H(\xi), H(\beta)_{1}, H_{2}$ hold, then the map $\lambda \rightarrow u_{\lambda}$ is nondecreasing and continuous from $\mathcal{L}=\left(-\infty, \widehat{\lambda}_{1}\right)$ into $C^{1}(\bar{\Omega})$.

In fact, by strengthening hypothesis $H(\beta)_{1}$ (since we will use Proposition 4) and with an additional condition on the perturbation $f(z, \cdot)$ we can improve the monotonicity property of the maps $\lambda \rightarrow \bar{u}_{\lambda}$ in Proposition 11 and of the map $\lambda \rightarrow u_{\lambda}$ in Proposition 12.

So, we introduce the following conditions on the functions $\beta(z)$ and $f(z, x)$ : $H(\beta)_{2}: \beta \in C^{0, \alpha}(\partial \Omega)$ with $\alpha \in(0,1)$ and $\beta(z)>0$ for all $z \in \partial \Omega$.

$H_{3}: f: \Omega \times \mathbb{R} \rightarrow \mathbb{R}$ is a Carathéodory function s.t. for a.a. $z \in \Omega, f(z, 0)=0$, $f(z, x)>0$ for all $x>0$, hypotheses $H_{3}$ (i), (ii), (iii) are the same as the corresponding hypotheses $H_{1}(\mathrm{i})$, (ii), (iii) and

(iv) for every $\rho>0$, there exists $\widehat{\xi}_{\rho}>0$ s.t. for a.a. $z \in \Omega$ the function $x \rightarrow$ $f(z, x)+\widehat{\xi}_{\rho} x^{p-1}$ is nondecreasing on $[0, \rho]$.

We also introduce a corresponding strengthening of hypotheses $H_{2}$.

$H_{4}: f: \Omega \times \mathbb{R} \rightarrow \mathbb{R}$ is a Carathéodory function s.t. for a.a. $z \in \Omega, f(z, 0)=0$, $f(z, x)>0$ for all $x>0$, hypotheses $H_{4}(\mathrm{i})$, (ii), (iii), (iv) are the same as the corresponding hypotheses $\mathrm{H}_{2}$ (i), (ii), (iii), (iv) and

(v) for every $\rho>0$, there exists $\widehat{\xi}_{\rho}>0$ s.t. for a.a. $z \in \Omega$, the function $x \rightarrow$ $f(z, x)+\widehat{\xi}_{\rho} x^{p-1}$ is nondecreasing on $[0, \rho]$.

Proposition 14 If hypotheses $H(\xi), H(\beta)_{2}, H_{3}$ hold, then the map $\lambda \rightarrow \bar{u}_{\lambda}$ is strictly increasing from $\mathcal{L}=\left(-\infty, \widehat{\lambda}_{1}\right)$ into $C^{1}(\bar{\Omega})$ in the sense that $\lambda<\mu \Rightarrow \bar{u}_{\mu}-\bar{u}_{\lambda} \in$ int $\widehat{C}_{+}$with $D_{0}=\left\{z \in \partial \Omega: \bar{u}_{\mu}(z)=\bar{u}_{\lambda}(z)\right\}$.

Proof Let $\lambda, \mu \in \mathcal{L}=\left(-\infty, \widehat{\lambda}_{1}\right)$ with $\lambda<\mu$. From Proposition 11 we know that

$$
\bar{u}_{\lambda} \leq \bar{u}_{\mu}
$$


Let $\rho=\left\|\bar{u}_{\mu}\right\|_{\infty}$ and let $\widehat{\xi}_{\rho}>0$ be as postulated by hypothesis $H_{3}$ (iv). We set

$$
\widetilde{\xi}_{\rho}=\widehat{\xi}_{\rho}+\max \{-\mu, 0\} .
$$

For $\delta>0$ we define $\bar{u}_{\lambda}^{\delta}=\bar{u}_{\lambda}+\delta \in D_{+}$. We have

$$
\begin{aligned}
- & \Delta_{p} \bar{u}_{\lambda}^{\delta}+\left(\xi(z)+\widetilde{\xi}_{\rho}\right)\left(\bar{u}_{\lambda}^{\delta}\right)^{p-1} \\
& \leq-\Delta_{p} \bar{u}_{\lambda}+\left(\xi(z)+\widetilde{\xi}_{\rho}\right) \bar{u}_{\lambda}^{p-1}+\chi(\delta) \quad \text { with } \chi(\delta) \rightarrow 0^{+} \text {as } \delta \rightarrow 0^{+} \\
& =\lambda \bar{u}_{\lambda}^{p-1}+f\left(z, \bar{u}_{\lambda}\right)+\widetilde{\xi}_{\rho} \bar{u}_{\lambda}^{p-1}+\chi(\delta) \\
& =\mu \bar{u}_{\lambda}^{p-1}+f\left(z, \bar{u}_{\lambda}\right)+\widetilde{\xi}_{\rho} \bar{u}_{\lambda}^{p-1}-(\mu-\lambda) \bar{u}_{\lambda}^{p-1}+\chi(\delta) .
\end{aligned}
$$

Note that if $\mu<0$, then $\widetilde{\xi}_{\rho}=\widehat{\xi}_{\rho}+|\mu|$ and we have

$$
\begin{aligned}
0 & \leq\left[f\left(z, \bar{u}_{\mu}\right)+\widehat{\xi}_{\rho} \bar{u}_{\mu}^{p-1}-\left(f\left(z, \bar{u}_{\lambda}\right)+\widehat{\xi}_{\rho} \bar{u}_{\lambda}^{p-1}\right)\right]+(|\mu|+\mu)\left(\bar{u}_{\mu}^{p-1}-\bar{u}_{\lambda}^{p-1}\right) \\
& \Leftrightarrow \mu \bar{u}_{\lambda}^{p-1}+f\left(z, \bar{u}_{\lambda}\right)+\widetilde{\xi}_{\rho} \bar{u}_{\lambda}^{p-1} \leq \mu \bar{u}_{\mu}^{p-1}+f\left(z, \bar{u}_{\mu}\right)+\widetilde{\xi}_{\rho} \bar{u}_{\mu}^{p-1} .
\end{aligned}
$$

If $\mu \geq 0$, then $\widetilde{\xi}_{\rho}=\widehat{\xi}_{\rho}$ and using hypothesis $H_{3}$ (iv) we have

$$
\mu \bar{u}_{\lambda}^{p-1}+f\left(z, \bar{u}_{\lambda}\right)+\widehat{\xi}_{\rho} \bar{u}_{\lambda}^{p-1} \leq \mu \bar{u}_{\mu}^{p-1}+f\left(z, \bar{u}_{\mu}\right)+\widehat{\xi}_{\rho} \bar{u}_{\mu}^{p-1} .
$$

Returning to (39), we have

$$
\begin{aligned}
- & \Delta_{p} \bar{u}_{\lambda}^{\delta}+\left(\xi(z)+\widetilde{\xi}_{\rho}\right)\left(\bar{u}_{\lambda}^{\delta}\right)^{p-1} \\
& \leq \mu \bar{u}_{\mu}^{p-1}+f\left(z, \bar{u}_{\mu}\right)+\widetilde{\xi}_{\rho} \bar{u}_{\mu}^{p-1}-(\mu-\lambda) \bar{u}_{\lambda}^{p-1}+\chi(\delta) \\
& =-\Delta_{p} \bar{u}_{\mu}+\widetilde{\xi}_{\rho} \bar{u}_{\mu}^{p-1}-(\mu-\lambda) \bar{u}_{\lambda}^{p-1}+\chi(\delta) .
\end{aligned}
$$

Since $\mu>\lambda$ and $\bar{u}_{\lambda} \in D_{+}$, we have

$$
0<\widehat{m} \leq(\mu-\lambda) \bar{u}_{\lambda}(z)^{p-1} \text { for all } z \in \bar{\Omega} .
$$

Then since $\chi(\delta) \rightarrow 0^{+}$as $\delta \rightarrow 0^{+}$, for $\delta>0$ small we have

$$
\widehat{m}-\chi(\delta)>0 \text {. }
$$

Using this in (40) we have

$$
\begin{aligned}
& -\Delta_{p} \bar{u}_{\lambda}^{\delta}+\left(\xi(z)+\widetilde{\xi}_{\rho}\right)\left(\bar{u}_{\lambda}^{\delta}\right)^{p-1}<-\Delta_{p} \bar{u}_{\mu}+\left(\xi(z)+\widetilde{\xi}_{\rho}\right) \bar{u}_{\mu} \\
& \text { for a.a. } z \in \Omega, \text { all } \delta>0 \text { small, }
\end{aligned}
$$$$
\Rightarrow \bar{u}_{\mu}-\bar{u}_{\lambda} \in \operatorname{int} \widehat{C}_{+}, \quad \text { (see Proposition } 4 \text { and the Remark that follows). }
$$

In this case in the definition of $\widehat{C}_{+}, D_{0}=\left\{z \in \partial \Omega: \bar{u}_{\mu}(z)=\bar{u}_{\lambda}(z)\right\}$. 
Similarly we have:

Proposition 15 If hypotheses $H(\xi), H(\beta)_{2}, H_{4}$ hold, then the map $\lambda \rightarrow \bar{u}_{\lambda}$ is strictly increasing from $\mathcal{L}=\left(-\infty, \widehat{\lambda}_{1}\right)$ into $C^{1}(\bar{\Omega})$.

The next theorem summarizes the situation for problem $\left(P_{\lambda}\right)$ when the perturbation $f(z, \cdot)$ is $(p-1)$-sublinear.

Theorem 1 We have:

1. If hypotheses $H(\xi), H(\beta)_{1}, H_{1}$ hold, then

(a) for all $\lambda \geq \widehat{\lambda}_{1}$ problem $\left(P_{\lambda}\right)$ has no positive solution;

(b) for all $\lambda<\widehat{\lambda}_{1}$ problem $\left(P_{\lambda}\right)$ has at least one positive solution and it admits a smallest positive solution $\bar{u}_{\lambda} \in D_{+}$;

(c) the map $\lambda \rightarrow \bar{u}_{\lambda}$ from $\mathcal{L}=\left(-\infty, \widehat{\lambda}_{1}\right)$ into $C^{1}(\bar{\Omega})$ is nondecreasing (that is, if $\lambda \leq \mu$, then $\bar{u}_{\lambda} \leq \bar{u}_{\mu}$ ) and left continuous.

2. If hypotheses $H(\xi), H(\beta)_{2}, H_{3}$ hold, then the map $\lambda \rightarrow \bar{u}_{\lambda}$ from $\mathcal{L}=\left(-\infty, \widehat{\lambda}_{1}\right)$ into $C^{1}(\bar{\Omega})$ is strictly increasing as in Proposition 14 .

3. If hypotheses $H(\xi), H(\beta)_{1}, H_{2}$ hold and $\lambda<\widehat{\lambda}_{1}$, then problem $\left(P_{\lambda}\right)$ has a unique solution $u_{\lambda} \in D_{+}$and the map $\lambda \rightarrow u_{\lambda}$ from $\mathcal{L}=\left(-\infty, \widehat{\lambda}_{1}\right)$ into $C^{1}(\bar{\Omega})$ is nondecreasing and continuous.

4. If hypotheses $H(\xi), H(\beta)_{2}, H_{4}$ hold, then the solution map $\lambda \rightarrow \bar{u}_{\lambda}$ from $\mathcal{L}=$ $\left(-\infty, \widehat{\lambda}_{1}\right)$ into $C^{1}(\bar{\Omega})$ is strictly increasing.

\section{$4(p-1)$-superlinear perturbation}

In this section we consider the case where the perturbation $f(z, \cdot)$ is $(p-1)$-superlinear. In this case uniqueness of the solution fails and the problem exhibits a bifurcation-type behaviour, namely there are no positive solutions for all $\lambda \geq \widehat{\lambda}_{1}$ and there are at least two positive solutions for $\lambda<\widehat{\lambda}_{1}$.

The new hypotheses on the perturbation term $f(z, x)$ are the following:

$H_{5}: f: \Omega \times \mathbb{R} \rightarrow \mathbb{R}$ is a Carathéodory function s.t. for a.a. $z \in \Omega f(z, 0)=0$, $f(z, x) \geq 0$ for all $x>0$, there exist $\Omega_{0} \subseteq \Omega$ with $\left|\Omega_{0}\right|_{N}>0$ s.t. $f(z, x)>0$ for all $z \in \Omega_{0}$, all $x>0$ and

(i) $f(z, x) \leq a(z)\left(1+x^{r-1}\right)$ for a.a. $z \in \Omega$, all $x \geq 0$, with $a \in L^{\infty}(\Omega)_{+}$, $r \in\left(p, p^{*}\right)$

(ii) if $F(z, x)=\int_{0}^{x} f(z, s) d s$, then

$$
\lim _{x \rightarrow+\infty} \frac{F(z, x)}{x^{p}}=+\infty \text { uniformly for a.a. } z \in \Omega
$$

and there exists $\tau \in\left(\max \left\{1,(r-p) \frac{N}{p}\right\}, p^{*}\right)$ s.t.

$$
0<\widetilde{\xi} \leq \liminf _{x \rightarrow+\infty} \frac{f(z, x) x-p F(z, x)}{x^{\tau}} \text { uniformly for a.a. } z \in \Omega ;
$$


(iii) $\lim _{x \rightarrow 0^{+}} \frac{f(z, x)}{x^{p-1}}=0$ uniformly for a.a. $z \in \Omega$.

Remark 4 As we did for the "sublinear" case, since we are looking for positive solutions and the above hypotheses concern the positive semiaxis, without any loss of generality, we may assume that $f(z, x)=0$ for a.a. $z \in \Omega$, all $x<0$. Hypothesis $H_{5}$ (ii) implies that for a.a. $z \in \Omega f(z, \cdot)$ is $(p-1)$-superlinear. However, note that we do not use the usual in such cases "Ambrosetti-Rabinowitz condition" (the AR-condition for short, unilateral version since we are looking for positive solutions), which says that there exist $q>p$ and $M>0$ s.t.

$$
\begin{aligned}
& 0<q F(z, x) \leq f(z, x) x \text { for a.a. } z \in \Omega, \text { all } x \geq M, \\
& 0<\operatorname{ess} \inf _{\Omega} F(\cdot, M)
\end{aligned}
$$

(see Ambrosetti-Rabinowitz [3] and Mugnai [15]). Integrating (41) and using (42) we obtain

$$
c_{13} x^{q} \leq F(z, x) \text { for a.a. } z \in \Omega \text {, all } x \geq M \text {, some } c_{13}>0 \text {. }
$$

Hence from (41) and (43) we infer that near $+\infty, f(z, \cdot)$ exhibits at least $(q-1)$ polynomial growth. Our hypothesis $H_{5}$ (ii) is more general. Indeed, suppose that the AR-condition holds. We may assume that $q>\max \left\{1,(r-p) \frac{N}{p}\right\}$. We have

$$
\begin{aligned}
& \frac{f(z, x) x-p F(z, x)}{x^{q}}=\frac{f(z, x) x-q F(z, x)}{x^{q}}+(q-p) \frac{F(z, x)}{x^{q}} \\
& \geq(q-p) \frac{F(z, x)}{x^{q}} \quad(\text { see }(41)) \\
& \geq(q-p) c_{13}>0 \quad(\text { see }(43)), \\
& \Rightarrow \liminf _{x \rightarrow+\infty} \frac{f(z, x) x-p F(z, x)}{x^{q}} \geq(q-p) c_{13}>0 \quad \text { uniformly for a.a. } z \in \Omega, \\
& \Rightarrow \text { hypothesis } H_{5} \text { (ii) holds. }
\end{aligned}
$$

The function

$$
f(x)=x^{p-1} \ln (1+x) \text { for all } x \geq 0
$$

satisfies hypotheses $H_{5}$, but not the AR-condition (see (41)).

From Propositions 5 and 6 we have

$$
\begin{aligned}
& S(\lambda) \subseteq D_{+} \text {for all } \lambda \in \mathbb{R}, \\
& S(\lambda)=\varnothing \text { for all } \lambda \geq \widehat{\lambda}_{1} .
\end{aligned}
$$

It follows that $\mathcal{L} \subseteq\left(-\infty, \widehat{\lambda}_{1}\right)$. In the next proposition we show that equality holds. Proposition 16 If hypotheses $H(\xi), H(\beta)_{1}, H_{5}$ hold, then $\mathcal{L}=\left(-\infty, \widehat{\lambda}_{1}\right)$. 
Proof We fix $\lambda \in\left(-\infty, \widehat{\lambda}_{1}\right)$ and consider the Carathéodory function $k_{\lambda}(z, x)$ defined by

$$
k_{\lambda}(z, x)= \begin{cases}0 & \text { if } x \leq 0 \\ \lambda x^{p-1}+f(z, x) & \text { if } 0<x\end{cases}
$$

We set $K_{\lambda}(z, x)=\int_{0}^{x} k_{\lambda}(z, x) d s$ and consider the $C^{1}$-functional $w_{\lambda}: W^{1, p}(\Omega) \rightarrow$ $\mathbb{R}$ defined by

$$
w_{\lambda}(u)=\frac{1}{p} \vartheta(u)+\frac{\eta}{p}\left\|u^{-}\right\|_{p}^{p}-\int_{\Omega} K_{\lambda}(z, u) d z \text { for all } u \in W^{1, p}(\Omega) .
$$

As before $\eta>\|\xi\|_{\infty}$. Hypotheses $H_{5}(\mathrm{i})$, (iii) imply that given $\varepsilon>0$, we can find $c_{14}=c_{14}(\varepsilon)>0$ s.t.

$$
F(z, x) \leq \frac{\varepsilon}{p} x^{p}+c_{14} x^{r} \text { for a.a. } z \in \Omega, \text { all } x \geq 0 .
$$

Choosing $\varepsilon \in\left(0, \widehat{\lambda}_{1}-\lambda\right)$ (recall $\left.\lambda<\widehat{\lambda}_{1}\right)$, for every $u \in W^{1, p}(\Omega)$ we have

$$
\begin{aligned}
w_{\lambda}(u) \geq & \frac{1}{p}\left[\vartheta\left(u^{-}\right)+\eta\left\|u^{-}\right\|_{p}^{p}\right]+\frac{1}{p} \vartheta\left(u^{+}\right)-\frac{\lambda+\varepsilon}{p}\left\|u^{+}\right\|_{p}^{p}-c_{14}\left\|u^{+}\right\|_{r}^{r} \\
& \quad(\text { see }(44) \text { and }(45)) . \\
\geq & c_{15}\|u\|^{p}-c_{16}\|u\|^{r} \text { for some } c_{15}, c_{16}>0, \\
& \quad\left(\text { use Lemma } 1 \text { and recall } \eta>\|\xi\|_{\infty}\right) .
\end{aligned}
$$

Since $p<r$, from (46) we infer that $u=0$ is a strict local minimizer of $w_{\lambda}$. So, we can find $\rho \in(0,1)$ small s.t.

$$
w_{\lambda}(0)=0<\inf \left[w_{\lambda}:\|u\|=\rho\right]=m_{\rho}^{\lambda}
$$

(see Aizicovici-Papageorgiou-Staicu [1], proof of Proposition 29).

Hypothesis $H_{5}$ (ii) implies that

$$
w_{\lambda}\left(t \widehat{u}_{1}\right) \rightarrow-\infty \quad \text { as } t \rightarrow+\infty .
$$

Claim: $w_{\lambda}$ satisfies the $C$-condition.

Let $\left\{u_{n}\right\}_{n \geq 1} \subseteq W^{1, p}(\Omega)$ be a sequence s.t.

$$
\begin{aligned}
& \left|w_{\lambda}\left(u_{n}\right)\right| \leq M_{1} \text { for some } M_{1}>0, \text { all } n \in \mathbb{N}, \\
& \left(1+\left\|u_{n}\right\|\right) w_{\lambda}^{\prime}\left(u_{n}\right) \rightarrow 0 \text { in } W^{1, p}(\Omega)^{*} \text { as } n \rightarrow+\infty
\end{aligned}
$$

From (50) we have

$$
\left.\left|\left\langle A\left(u_{n}\right), h\right\rangle+\int_{\Omega} \xi(z)\right| u_{n}\right|^{p-2} u_{n} h d z+\int_{\partial \Omega} \beta(z)\left|u_{n}\right|^{p-2} u_{n} h d \sigma
$$




$$
\begin{aligned}
& -\eta \int_{\Omega}\left(u_{n}^{-}\right)^{p-1} h d \sigma-\int_{\Omega} k_{\lambda}\left(z, u_{n}\right) h d z \mid \\
& \leq \frac{\varepsilon_{n}\|h\|}{1+\left\|u_{n}\right\|} \text { for all } h \in W^{1, p}(\Omega) \text { with } \varepsilon_{n} \rightarrow 0^{+}
\end{aligned}
$$

In (51) we choose $h=-u_{n}^{-} \in W^{1, p}(\Omega)$. Using (44) we obtain

$$
\begin{aligned}
& \left|\vartheta\left(u_{n}^{-}\right)+\eta\left\|u_{n}^{-}\right\|_{p}^{p}\right| \leq \varepsilon_{n} \text { for all } n \in \mathbb{N} \\
& \quad \Rightarrow c_{17}\left\|u_{n}^{-}\right\|^{p} \leq \varepsilon_{n} \quad \text { for all } n \in \mathbb{N}, \text { some } c_{17}>0\left(\text { recall that } \eta>\|\xi\|_{\infty}\right) \\
& \quad \Rightarrow u_{n}^{-} \rightarrow 0 \text { in } W^{1, p}(\Omega)
\end{aligned}
$$

From (49), (52) and (44) it follows that

$$
\vartheta\left(u_{n}^{+}\right)-\int_{\Omega}\left[\lambda\left(u_{n}^{+}\right)^{p}+p F\left(z, u_{n}^{+}\right)\right] d z \leq M_{2} \text { for some } M_{2}>0, \text { all } n \in \mathbb{N} \text {. }
$$

In (51) we choose $h=u_{n}^{+} \in W^{1, p}(\Omega)$. Then

$$
-\vartheta\left(u_{n}^{+}\right)+\int_{\Omega}\left[\lambda\left(u_{n}^{+}\right)^{p}+f\left(z, u_{n}^{+}\right) u_{n}^{+}\right] d z \leq \varepsilon_{n} \quad \text { for all } n \in \mathbb{N} .
$$

Adding (53) and (54) we obtain

$$
\int_{\Omega}\left[f\left(z, u_{n}^{+}\right) u_{n}^{+}-p F\left(z, u_{n}^{+}\right)\right] d z \leq M_{3} \text { for some } M_{3}>0 \text {, all } n \in \mathbb{N} \text {. }
$$

Hypotheses $H_{5}(\mathrm{i})$, (ii) imply that we can find $\widetilde{\xi}_{0} \in(0, \widetilde{\xi})$ and $c_{18}>0$ s.t.

$$
\widetilde{\xi}_{0} x^{\tau}-c_{18} \leq f(z, x) x-p F(z, x) \text { for a.a. } z \in \Omega \text {, all } x \geq 0
$$

Using (56) in (55), we infer that

$$
\left\{u_{n}^{+}\right\}_{n \geq 1} \subseteq L^{\tau}(\Omega) \text { is bounded. }
$$

First suppose that $N>p$. Clearly in hypothesis $H_{5}$ (ii), we can always assume that $\tau<r<p^{*}$ (recall that $p^{*}=+\infty$ if $p \geq N$ ). Let $t \in(0,1)$ be such that

$$
\frac{1}{r}=\frac{1-t}{\tau}+\frac{t}{p^{*}}
$$

From the interpolation inequality (see, for example, Gasiński-Papageorgiou [8] (p. 905)), we have

$$
\begin{aligned}
& \left\|u_{n}^{+}\right\|_{r} \leq\left\|u_{n}^{+}\right\|_{\tau}^{1-t}\left\|u_{n}^{+}\right\|_{p^{*}}^{t}, \\
& \quad \Rightarrow\left\|u_{n}^{+}\right\|_{r}^{r} \leq M_{4}\left\|u_{n}^{+}\right\|^{t r} \text { for some } M_{4}>0, \text { all } n \in \mathbb{N}
\end{aligned}
$$


(see (57) and use the Sobolev embedding theorem).

In (51) we choose $h=u_{n}^{+} \in W^{1, p}(\Omega)$. Then

$$
\begin{aligned}
& \vartheta\left(u_{n}^{+}\right)-\int_{\Omega}\left[\lambda\left(u_{n}^{+}\right)^{p}+f\left(z, u_{n}^{+}\right) u_{n}^{+}\right] d z \leq \varepsilon_{n} \text { for all } n \in \mathbb{N}(\text { see }(44)), \\
& \Rightarrow \vartheta\left(u_{n}^{+}\right) \leq c_{19}\left(1+\left\|u_{n}^{+}\right\|_{r}^{r}\right) \quad \text { for some } c_{19}>0 \text {, all } n \in \mathbb{N} \\
& \quad\left(\text { see hypothesis } H_{5}(\mathrm{i}) \text { and recall that } r>p\right), \\
& \Rightarrow \vartheta\left(u_{n}^{+}\right) \leq c_{20}\left(1+\left\|u_{n}^{+}\right\|^{t r}\right) \text { for some } c_{20}>0 \text {, all } n \in \mathbb{N}(\text { see (59)). }
\end{aligned}
$$

From hypothesis $H_{5}(\mathrm{i})$ we see that we can always take $r \in\left(p, p^{*}\right)$ close to $p^{*}$ and as $r \rightarrow\left(p^{*}\right)^{-}$, we have $\tau>p$. So, there is no loss of generality in assuming that $\tau>p$. Then from (60) and (57), we have

$$
\begin{aligned}
& \vartheta\left(u_{n}^{+}\right)+\eta\left\|u_{n}^{+}\right\|_{p}^{p} \leq c_{21}\left(1+\left\|u_{n}^{+}\right\|^{t r}\right) \text { for some } c_{21}>0, \text { all } n \in \mathbb{N}, \\
& \left.\quad \Rightarrow\left\|u_{n}^{+}\right\|^{p} \leq c_{22}\left(1+\left\|u_{n}^{+}\right\|^{t r}\right) \text { for some } c_{22}>0 \text {, all } n \in \mathbb{N} \text { (recall that } \eta>\|\xi\|_{\infty}\right) .
\end{aligned}
$$

From hypothesis $H_{5}(\mathrm{ii})$ and (58) we see that

$$
\begin{aligned}
t r & <p \\
& \left.\Rightarrow\left\{u_{n}^{+}\right\}_{n \geq 1} \subseteq W^{1, p}(\Omega) \quad \text { is bounded (see }(61)\right), \\
& \left.\Rightarrow\left\{u_{n}\right\}_{n \geq 1} \subseteq W^{1, p}(\Omega) \quad \text { is bounded (see }(52)\right)
\end{aligned}
$$

If $N \leq p$, then $p^{*}=+\infty$, while the Sobolev embedding theorem says that $W^{1, p}(\Omega) \hookrightarrow L^{q}(\Omega)$ for all $q \in[1,+\infty)$. Let $q>r>\tau$ and choose $t \in(0,1)$ s.t.

$$
\begin{aligned}
\frac{1}{r} & =\frac{1-t}{\tau}+\frac{t}{q}, \\
& \Rightarrow t r=\frac{q(r-\tau)}{q-\tau} .
\end{aligned}
$$

Note that

$$
\frac{q(r-\tau)}{q-\tau} \rightarrow r-\tau \text { as } q \rightarrow p^{*}=+\infty
$$

Since by hypothesis $H_{5}$ (ii) we have $r-\tau<p$ (recall $N \leq p$ ), for the previous argument (case $N \leq p$ ) to work, we use $q>r$ big s.t. $t r<p$ (see (63), (64)). Then again we conclude that (62) holds. Because of (62) we may assume that

$$
u_{n} \stackrel{w}{\rightarrow} u \text { in } W^{1, p}(\Omega) \text { and } u_{n} \rightarrow u \text { in } L^{p}(\Omega) \text { and in } L^{p}(\partial \Omega) .
$$


In (51) we choose $h=u_{n}-u \in W^{1, p}(\Omega)$, pass to the limit as $n \rightarrow+\infty$ and use (65). Then we have

$$
\begin{aligned}
& \lim _{n \rightarrow+\infty}\left\langle A\left(u_{n}\right), u_{n}-u\right\rangle=0, \\
& \quad \Rightarrow u_{n} \rightarrow u \text { in } W^{1, p}(\Omega) \quad(\text { see Proposition } 2), \\
& \Rightarrow w_{\lambda} \text { satisfies the } C \text {-condition. }
\end{aligned}
$$

This proves the Claim.

Then (47), (48) and the Claim permit the use of the mountain pass theorem (see, for example, Gasiński-Papageorgiou [8] (p. 648)). So, we can find $u_{\lambda} \in W^{1, p}(\Omega)$ s.t.

$$
u_{\lambda} \in K_{w_{\lambda}}=\left\{v \in W^{1, p}(\Omega): w_{\lambda}^{\prime}(v)=0\right\} \quad \text { and } \quad w_{\lambda}(0)=0<m_{\rho}^{\lambda} \leq w_{\lambda}\left(u_{\lambda}\right)
$$

From (66) it follows that $u_{\lambda} \neq 0$ and $u_{\lambda} \in S(\lambda) \subseteq D_{+}$(see Proposition 5). Therefore $\mathcal{L}=\left(-\infty, \widehat{\lambda}_{1}\right)$.

In fact as we did in the "sublinear" case, we can produce the minimal positive solution for problem $\left(P_{\lambda}\right), \lambda<\widehat{\lambda}_{1}$.

Proposition 17 If hypotheses $H(\xi), H(\beta)_{1}, H_{5}$ hold and $\lambda \in \mathcal{L}=\left(-\infty, \widehat{\lambda}_{1}\right)$, then problem $\left(P_{\lambda}\right)$ has a smallest positive solution $\bar{u}_{\lambda} \in D_{+}$.

Proof We argue as in the proof of Proposition 10. Recall that $S(\lambda)$ is downward directed (see Filippakis-Papageorgiou [5]). Using Lemma 3.10 of Hu-Papageorgiou [9] (p. 178), we can find a decreasing sequence $\left\{u_{n}\right\}_{n \geq 1} \subseteq S(\lambda)$ s.t.

$$
\inf S(\lambda)=\inf _{n \geq 1} u_{n}
$$

We have

$$
\left\langle A\left(u_{n}\right), h\right\rangle+\int_{\Omega} \xi(z) u_{n}^{p-1} h d z+\int_{\partial \Omega} \beta(z) u_{n}^{p-1} h d \sigma=\int_{\Omega}\left[\lambda u_{n}^{p-1}+f\left(z, u_{n}\right)\right] h d z
$$

for all $h \in W^{1, p}(\Omega)$, all $n \in \mathbb{N}$.

In (67) we choose $h=u_{n} \in W^{1, p}(\Omega)$, we obtain

$$
\vartheta\left(u_{n}\right)=\lambda\left\|u_{n}\right\|_{p}^{p}+\int_{\Omega} f\left(z, u_{n}\right) u_{n} d z \quad \text { for all } n \in \mathbb{N} .
$$

Recall that

$$
0 \leq u_{n} \leq u_{1} \in D_{+} \quad \text { for all } n \in \mathbb{N} \text {. }
$$


From (68) to (69) it follows that

$$
\left.\left\{u_{n}\right\}_{n \geq 1} \subseteq W^{1, p}(\Omega) \text { is bounded (see hypotheses } H(\xi), H(\beta)_{1}\right) .
$$

So, we may assume that

$$
u_{n} \stackrel{w}{\rightarrow} \bar{u}_{\lambda} \text { in } W^{1, p}(\Omega) \text { and } \quad u_{n} \rightarrow \bar{u}_{\lambda} \text { in } L^{p}(\Omega) \text { and in } L^{p}(\partial \Omega)
$$

In (67) we choose $h=u_{n}-\bar{u}_{\lambda} \in W^{1, p}(\Omega)$, pass to the limit as $n \rightarrow+\infty$ and use (70). Then

$$
\begin{aligned}
& \lim _{n \rightarrow+\infty}\left\langle A\left(u_{n}\right), u_{n}-\bar{u}_{\lambda}\right\rangle=0, \\
& \quad \Rightarrow u_{n} \rightarrow \bar{u}_{\lambda} \text { in } W^{1, p}(\Omega) \quad(\text { see Proposition } 2) .
\end{aligned}
$$

Passing to the limit as $n \rightarrow+\infty$ in (67) and using (71), we obtain

$$
\begin{aligned}
& \left\langle A\left(\bar{u}_{\lambda}\right), h\right\rangle+\int_{\Omega} \xi(z) \bar{u}_{\lambda}^{p-1} h d z+\int_{\partial \Omega} \beta(z) \bar{u}_{\lambda}^{p-1} h d \sigma \\
& =\int_{\Omega}\left[\lambda \bar{u}_{\lambda}^{p-1}+f\left(z, \bar{u}_{\lambda}\right)\right] h d z \quad \text { for all } h \in W^{1, p}(\Omega), \\
& \Rightarrow \bar{u}_{\lambda} \text { is a nonnegative solution of problem }\left(P_{\lambda}\right) .
\end{aligned}
$$

If we can show that $\bar{u}_{\lambda} \neq 0$, then $\bar{u}_{\lambda} \in S(\lambda) \subseteq D_{+}$. Arguing by contradiction, suppose that $\bar{u}_{\lambda}=0$. Then

$$
\left\|u_{n}\right\| \rightarrow 0 \quad(\operatorname{see}(71))
$$

We set $y_{n}=\frac{u_{n}}{\left\|u_{n}\right\|}, n \in \mathbb{N}$. Then for all $n \in \mathbb{N}$ we have $\left\|y_{n}\right\|=1, y_{n} \geq 0$. So, we may assume that

$$
y_{n} \stackrel{w}{\rightarrow} y \text { in } W^{1, p}(\Omega) \quad \text { and } \quad y_{n} \rightarrow y \text { in } L^{p}(\Omega) \text { and in } L^{p}(\partial \Omega) .
$$

From (67) we have

$$
\begin{aligned}
& \left\langle A\left(y_{n}\right), h\right\rangle+\int_{\Omega} \xi(z) y_{n}^{p-1} h d z+\int_{\partial \Omega} \beta(z) y_{n}^{p-1} h d \sigma \\
& \quad=\int_{\Omega}\left[\lambda y_{n}^{p-1}+\frac{N_{f}\left(u_{n}\right)}{\left\|u_{n}\right\|^{p-1}}\right] h d z \quad \text { for all } h \in W^{1, p}(\Omega), \text { all } n \in \mathbb{N} .
\end{aligned}
$$

Here $N_{f}(y)(\cdot)=f(\cdot, y(\cdot))$ for all $y \in W^{1, p}(\Omega)$. We set $\rho=\left\|u_{1}\right\|_{\infty}$. Hypotheses $H_{5}$ (i), (iii) imply that

$$
0 \leq f(z, x) \leq c_{23} x^{p-1} \quad \text { for a.a. } z \in \Omega, \text { all } x \in[0, \rho], \text { some } c_{23}>0,
$$




$$
\Rightarrow\left\{\frac{N_{f}\left(u_{n}\right)}{\left\|u_{n}\right\|^{p-1}}\right\}_{n \geq 1} \subseteq L^{p}(\Omega) \quad \text { is bounded. }
$$

Then by passing to a suitable subsequence if necessary and using hypothesis $H_{5}$ (iii), we have

$$
\frac{N_{f}\left(u_{n}\right)}{\left\|u_{n}\right\|^{p-1}} \stackrel{w}{\rightarrow} 0 \quad \text { in } L^{p}(\Omega)
$$

(see Aizicovici-Papageorgiou-Staicu [1], proof of Proposition 14).

In (73) we choose $h=y_{n}-y \in W^{1, p}(\Omega)$, pass to the limit as $n \rightarrow+\infty$ and use (72) and (74). Then

$$
\begin{aligned}
& \lim _{n \rightarrow+\infty}\left\langle A\left(y_{n}\right), y_{n}-y\right\rangle=0, \\
& \quad \Rightarrow y_{n} \rightarrow y \text { in } W^{1, p}(\Omega) \quad(\text { see Proposition } 2),\|y\|=1, y \geq 0 .
\end{aligned}
$$

So, if in (73) we pass to the limit as $n \rightarrow+\infty$ and use (74) and (75), then

$$
\begin{aligned}
& \langle A(y), h\rangle+\int_{\Omega} \xi(z) y^{p-1} h d z+\int_{\partial \Omega} \beta(z) y^{p-1} h d \sigma=\lambda \int_{\Omega} y^{p-1} h d z \\
& \quad \text { for all } h \in W^{1, p}(\Omega) .
\end{aligned}
$$

Choosing $h=y \in W^{1, p}(\Omega)$, we obtain

$$
\vartheta(y)=\lambda\|y\|_{p}^{p}<\widehat{\lambda}_{1}\|y\|_{p}^{p} \quad\left(\text { see }(75) \text { and recall } \lambda<\widehat{\lambda}_{1}\right)
$$

a contradiction to Proposition 1. Therefore

$$
\begin{aligned}
& \bar{u}_{\lambda} \neq 0 \\
& \quad \Rightarrow \bar{u}_{\lambda} \in S(\lambda) \text { and } \quad \bar{u}_{\lambda}=\inf S(\lambda) .
\end{aligned}
$$

As in the "sublinear" case, we have:

Proposition 18 If hypotheses $H(\xi), H(\beta)_{1}, H_{5}$ hold, then the map $\lambda \rightarrow \bar{u}_{\lambda}$ from $\mathcal{L}=\left(-\infty, \widehat{\lambda}_{1}\right)$ into $C^{1}(\bar{\Omega})$ is nondecreasing and left continuous.

Again by strengthening the conditions on the functions $\beta(\cdot)$ and $f(z, \cdot)$ we can improve the monotonicity of the map $\lambda \rightarrow \bar{u}_{\lambda}$.

The new hypotheses on the perturbation $f(z, x)$ are the following:

$H_{6}: f: \Omega \times \mathbb{R} \rightarrow \mathbb{R}$ is a Carathéodory function s.t. for a.a. $z \in \Omega, f(z, 0)=0$, $f(z, x) \geq 0$ for all $x>0$, there exists $\Omega_{0} \subseteq \Omega$ with $f(z, x)>0$ for all $z \in \Omega_{0}$, all $x>0$, hypotheses $H_{6}$ (i), (ii), (iii) are the same as the corresponding hypotheses $H_{5}(\mathrm{i})$, (ii), (iii) and 
(iv) for every $\rho>0$, there exists $\widehat{\xi}_{\rho}>0$ s.t. for a.a. $z \in \Omega$, the function

$$
x \rightarrow f(z, x)+\widehat{\xi}_{\rho} x^{p-1}
$$

is nondecreasing on $[0, \rho]$.

Proposition 19 If hypotheses $H(\xi), H(\beta)_{2}, H_{6}$ hold, then the map $\lambda \rightarrow \bar{u}_{\lambda}$ from $\mathcal{L}=\left(-\infty, \widehat{\lambda}_{1}\right)$ into $C^{1}(\bar{\Omega})$ is strictly decreasing.

In fact under these stronger conditions on $\beta(z)$ and $f(z, x)$, we can produce a second positive solution for problem $\left(P_{\lambda}\right)$, when $\lambda \in \mathcal{L}=\left(-\infty, \widehat{\lambda}_{1}\right)$.

Proposition 20 If hypotheses $H(\xi), H(\beta)_{2}, H_{6}$ hold and $\lambda \in \mathcal{L}=\left(-\infty, \widehat{\lambda}_{1}\right)$, then problem $\left(P_{\lambda}\right)$ admits at least two positive solutions

$$
u_{\lambda}, \widehat{u}_{\lambda} \in D_{+}, \quad u_{\lambda} \leq \widehat{u}_{\lambda}, \quad u_{\lambda} \neq \widehat{u}_{\lambda}
$$

Proof From Proposition 16 we already have a positive solution $u_{\lambda} \in D_{+}$. We may assume that $u_{\lambda}$ is the minimal positive solution, that is, $u_{\lambda}=\bar{u}_{\lambda}$ (see Proposition 17). We introduce the following Carathéodory function

$$
\zeta_{\lambda}(z, x)= \begin{cases}(\lambda+\eta) u_{\lambda}(z)^{p-1}+f\left(z, u_{\lambda}(z)\right) & \text { if } x \leq u_{\lambda}(z), \\ (\lambda+\eta) x^{p-1}+f(z, x) & \text { if } u_{\lambda}(z)<x,\end{cases}
$$

with $\eta>\|\xi\|_{\infty}$ as always. We set $Z_{\lambda}(z, x)=\int_{0}^{x} \zeta_{\lambda}(z, s) d s$ and consider the $C^{1}$ functional $j_{\lambda}: W^{1, p}(\Omega) \rightarrow \mathbb{R}$ defined by

$$
j_{\lambda}(u)=\frac{1}{p} \vartheta(u)+\frac{\eta}{p}\|u\|_{p}^{p}-\int_{\Omega} Z_{\lambda}(z, u) d z, \quad \text { for all } u \in W^{1, p}(\Omega) .
$$

From (76) it is clear that

$$
j_{\lambda}=w_{\lambda}+\xi_{\lambda}^{*} \text { with } \xi_{\lambda}^{*} \in \mathbb{R}
$$

with $w_{\lambda} \in C^{1}\left(W^{1, p}(\Omega)\right)$ as in the proof of Proposition 16. From (77) and the Claim in the proof of Proposition 16, it follows that

$$
j_{\lambda} \text { satisfies the } C \text {-condition. }
$$

Claim: We may assume that $u_{\lambda} \in D_{+}$is a local minimizer of $j_{\lambda}$.

Let $\lambda<\mu<\widehat{\lambda}_{1}$ and let $u_{\mu} \in S(\mu) \subseteq D_{+}$(see Proposition 16). We consider the following truncation of $\zeta_{\lambda}(z, \cdot)$

$$
\widehat{\zeta}_{\lambda}(z, x)= \begin{cases}\zeta(z, x) & \text { if } x \leq u_{\mu}(z) \\ \zeta\left(z, u_{\mu}(z)\right) & \text { if } u_{\mu}(z)<x\end{cases}
$$


Evidently this is a Carathéodory function. We set $\widehat{Z}_{\lambda}(z, x)=\int_{0}^{x} \widehat{\zeta}_{\lambda}(z, s) d s$ and consider the $C^{1}$-functional $\widehat{j_{\lambda}}: W^{1, p}(\Omega) \rightarrow \mathbb{R}$ defined by

$$
\widehat{j_{\lambda}}(u)=\frac{1}{p} \vartheta(u)+\frac{\eta}{p}\|u\|_{p}^{p}-\int_{\Omega} \widehat{Z}_{\lambda}(z, u) d z \quad \text { for all } u \in W^{1, p}(\Omega) .
$$

If $K_{\widehat{j}_{\lambda}}=\left\{u \in W^{1, p}(\Omega): \widehat{j}_{\lambda}^{\prime}(u)=0\right\}$, then we will show that

$$
K_{\widehat{j_{\lambda}}} \subseteq\left[u_{\lambda}, u_{\mu}\right]=\left\{u \in W^{1, p}(\Omega): u_{\lambda}(z) \leq u(z) \leq u_{\mu}(z) \text { for a.a. } z \in \Omega\right\} .
$$

So, let $u \in K_{\widehat{j}}$. Then

$$
\begin{aligned}
& \widehat{\hat{j}_{\lambda}}(u)=0 \\
& \quad \Rightarrow\langle A(u), h\rangle+\int_{\Omega}(\xi(z)+\eta)|u|^{p-2} u h d z+\int_{\partial \Omega} \beta(z)|u|^{p-2} u h d \sigma=\int_{\Omega} \widehat{\zeta}_{\lambda}(z, u) h d z
\end{aligned}
$$

for all $h \in W^{1, p}(\Omega)$.

In (80) we choose $h=\left(u_{\lambda}-u\right)^{+} \in W^{1, p}(\Omega)$. Then

$$
\begin{aligned}
& \left\langle A(u),\left(u_{\lambda}-u\right)^{+}\right\rangle+\int_{\Omega}(\xi(z)+\eta)|u|^{p-2} u\left(u_{\lambda}-u\right)^{+} d z+\int_{\partial \Omega} \beta(z)|u|^{p-2} u\left(u_{\lambda}-u\right)^{+} d \sigma \\
& =\int_{\Omega}\left[(\lambda+\eta) u_{\lambda}^{p-1}+f\left(z, u_{\lambda}\right)\right]\left(u_{\lambda}-u\right)^{+} d z \quad \text { (see (76) and (79)) } \\
& =\left\langle A\left(u_{\lambda}\right),\left(u_{\lambda}-u\right)^{+}\right\rangle+\int_{\Omega}(\xi(z)+\eta) u_{\lambda}^{p-1}\left(u_{\lambda}-u\right)^{+} d z \\
& \quad+\int_{\partial \Omega} \beta(z) u_{\lambda}^{p-1}\left(u_{\lambda}-u\right)^{+} d \sigma \quad\left(\text { since } u_{\lambda} \in S(\lambda)\right), \\
& \Rightarrow\left\langle A\left(u_{\lambda}\right)-A(u),\left(u_{\lambda}-u\right)^{+}\right\rangle+\int_{\Omega}(\xi(z)+\eta)\left(u_{\lambda}^{p-1}-|u|^{p-2} u\right)\left(u_{\lambda}-u\right)^{+} d z \\
& \quad+\int_{\partial \Omega} \beta(z)\left(u_{\lambda}^{p-1}-|u|^{p-2} u\right)\left(u_{\lambda}-u\right)^{+} d \sigma=0, \\
& \Rightarrow u_{\lambda} \leq u \quad\left(\text { recall that } \eta>\|\xi\|_{\infty} \text { and see hypothesis } H(\beta)\right) .
\end{aligned}
$$

Also in (80), we choose $h=\left(u-u_{\mu}\right)^{+} \in W^{1, p}(\Omega)$. Then

$$
\begin{aligned}
& \left\langle A(u),\left(u-u_{\mu}\right)^{+}\right\rangle+\int_{\Omega}(\xi(z)+\eta) u^{p-1}\left(u-u_{\mu}\right)^{+} d z+\int_{\partial \Omega} \beta(z) u^{p-1}\left(u-u_{\mu}\right)^{+} d \sigma \\
& \quad=\int_{\Omega}\left[(\lambda+\eta) u_{\mu}^{p-1}+f\left(z, u_{\mu}\right)\right]\left(u-u_{\mu}\right)^{+} d z \quad \text { (see (76), (79)) } \\
& \quad \leq \int_{\Omega}\left[(\mu+\eta) u_{\mu}^{p-1}+f\left(z, u_{\mu}\right)\right]\left(u-u_{\mu}\right)^{+} d z \quad(\text { since } \lambda<\mu) \\
& \quad=\left\langle A\left(u_{\mu}\right),\left(u-u_{\mu}\right)^{+}\right\rangle+\int_{\Omega}(\xi(z)+\eta) u_{\mu}^{p-1}\left(u-u_{\mu}\right)^{+} d z+\int_{\partial \Omega} \beta(z) u_{\mu}^{p-1}\left(u-u_{\mu}\right)^{+} d \sigma
\end{aligned}
$$




$$
\begin{aligned}
& \text { (since } \left.u_{\mu} \in S(\mu)\right), \\
& \Rightarrow\left\langle A(u)-A\left(u_{\mu}\right),\left(u-u_{\mu}\right)^{+}\right\rangle+\int_{\Omega}(\xi(z)+\eta)\left(u^{p-1}-u_{\mu}^{p-1}\right)\left(u-u_{\mu}\right)^{+} d z \\
& \quad+\int_{\partial \Omega} \beta(z)\left(u^{p-1}-u_{\mu}^{p-1}\right)\left(u-u_{\mu}\right)^{+} d \sigma \leq 0, \\
& \Rightarrow u \leq u_{\mu} .
\end{aligned}
$$

So, we have proved that

$$
\begin{aligned}
u & \in\left[u_{\lambda}, u_{\mu}\right], \\
& \Rightarrow K_{\widehat{j_{\lambda}}} \subseteq\left[u_{\lambda}, u_{\mu}\right] .
\end{aligned}
$$

Since $\eta>\|\xi\|_{\infty}$, from (76) and (79) it follows that $\widehat{j_{\lambda}}$ is coercive. Also, the Sobolev embedding theorem and the compactness of the trace map imply that $\widehat{j}_{\lambda}$ is sequentially weakly lower semicontinuous. So, from the Weierstrass-Tonelli theorem, we can find $\tilde{u}_{\lambda} \in W^{1, p}(\Omega)$ s.t.

$$
\begin{aligned}
& \widehat{j_{\lambda}}\left(\widetilde{u}_{\lambda}\right)=\inf \left[\widehat{j_{\lambda}}(u): u \in W^{1, p}(\Omega)\right] \\
& \Rightarrow \widetilde{u}_{\lambda} \in K_{\widehat{j_{\lambda}}} \subseteq\left[u_{\lambda}, u_{\mu}\right] \quad(\operatorname{see}(81)) .
\end{aligned}
$$

If $\tilde{u}_{\lambda} \neq u_{\lambda}$, then from (76), (79) and (82), we see that

$$
\tilde{u}_{\lambda} \in S(\lambda) \subseteq D_{+}, \quad u_{\lambda} \leq \tilde{u}_{\lambda}, \quad \tilde{u}_{\lambda} \neq u_{\lambda}
$$

So, this is the desired second solution of $\left(P_{\lambda}\right)$ and we are done.

Therefore, we assume that $\tilde{u}_{\lambda}=u_{\lambda}$. From Proposition 19, we have

$$
u_{\mu}-u_{\lambda} \in \operatorname{int} \widehat{C}_{+} \quad\left(\text { recall that } u_{\lambda}=\bar{u}_{\lambda}\right)
$$

From (76) and (79) it is clear that

$$
\left.\widehat{j_{\lambda}}\right|_{\left[0, u_{\mu}\right]}=\left.j_{\lambda}\right|_{\left[0, u_{\mu}\right]} \text {. }
$$

From this equality and (83) we infer that

$$
\begin{aligned}
& u_{\lambda} \text { is a local } C^{1}(\bar{\Omega}) \text {-minimizer of } j_{\lambda}, \\
& \left.\quad \Rightarrow u_{\lambda} \text { is a local } W^{1, p}(\Omega) \text {-minimizer of } j_{\lambda} \quad \text { (see Proposition } 3\right) .
\end{aligned}
$$

This proves the Claim.

In proving (81), we established that

$$
K_{j_{\lambda}} \subseteq\left[u_{\lambda}\right)=\left\{u \in W^{1, p}(\Omega): u_{\lambda}(z) \leq u(z) \text { for a.a. } z \in \Omega\right\} .
$$


We assume that $K_{j_{\lambda}}$ is finite or otherwise (84) implies that we already have a whole sequence of distinct positive solutions of $\left(P_{\lambda}\right)$, all bigger that $u_{\lambda}$, hence we are done. Then we can find $\rho \in(0,1)$ small s.t.

$$
j_{\lambda}\left(u_{\lambda}\right)<\inf \left[j_{\lambda}(u):\left\|u-u_{\lambda}\right\|=\rho\right]=m_{\rho}^{\lambda}
$$

(see Aizicovici-Papageorgiou-Staicu [1], proof of Proposition 29). Note that hypothesis $H_{6}$ (ii) and (76) imply that

$$
j_{\lambda}\left(t \widehat{u}_{1}\right) \rightarrow-\infty \quad \text { as } t \rightarrow+\infty
$$

From (78), (85) and (86) we see that we can apply the mountain pass theorem and find $\widehat{u}_{\lambda} \in W^{1, p}(\Omega)$ s.t.

$$
\widehat{u}_{\lambda} \in K_{j \lambda} \quad \text { and } \quad m_{\rho}^{\lambda} \leq j_{\lambda}\left(\widehat{u}_{\lambda}\right)
$$

From (76), (84), (85) and (87), we infer that

$$
\widehat{u}_{\lambda} \in S(\lambda) \subseteq D_{+}, \quad u_{\lambda} \leq \widehat{u}_{\lambda}, \quad \widehat{u}_{\lambda} \neq u_{\lambda}
$$

So, summarizing the situation for problem $\left(P_{\lambda}\right)$ when the perturbation $f(z, \cdot)$ is $(p-1)$-superlinear, we have the following theorem

Theorem 2 If hypotheses $H(\xi), H(\beta)_{2}, H_{6}$ hold, then

(a) for all $\lambda \geq \widehat{\lambda}_{1}$ problem $\left(P_{\lambda}\right)$ has no positive solution;

(b) for all $\lambda<\widehat{\lambda}_{1}$ problem $\left(P_{\lambda}\right)$ has at least two positive solutions

$$
u_{\lambda}, \widehat{u}_{\lambda} \in D_{+}, \quad u_{\lambda} \leq \widehat{u}_{\lambda}, \quad \widehat{u}_{\lambda} \neq u_{\lambda}
$$

(c) for all $\lambda<\widehat{\lambda}_{1}$ problem $\left(P_{\lambda}\right)$ has a smallest positive solution $\bar{u}_{\lambda} \in D_{+}$and the map $\lambda \rightarrow \bar{u}_{\lambda}$ from $\mathcal{L}=\left(-\infty, \widehat{\lambda}_{1}\right)$ into $C^{1}(\bar{\Omega})$ is strictly increasing and left continuous.

Funding Open access funding provided by Università degli Studi di Palermo within the CRUI-CARE Agreement.

\section{Compliance with ethical standards}

Conflict of interest The author declares that there is no conflict of interests regarding the publication of this article.

Open Access This article is licensed under a Creative Commons Attribution 4.0 International License, which permits use, sharing, adaptation, distribution and reproduction in any medium or format, as long as you give appropriate credit to the original author(s) and the source, provide a link to the Creative Commons licence, and indicate if changes were made. The images or other third party material in this article are included in the article's Creative Commons licence, unless indicated otherwise in a credit line to the material. If material is not included in the article's Creative Commons licence and your intended use is not permitted by statutory regulation or exceeds the permitted use, you will need to obtain permission directly from the copyright holder. To view a copy of this licence, visit http://creativecommons.org/licenses/by/4.0/. 


\section{References}

1. Aizicovici, S., Papageorgiou, N.S., Staicu, V.: Degree theory for operators of monotone type and nonlinear elliptic equations with inequality constraints. Mem. Amer. Math. Soc. 196(915) (2008). 70 $\mathrm{pp}$

2. Allegretto, W., Huang, Y.X.: A Picone's identity for the $p$-Laplacian and applications. Nonlinear Anal. 32(7), 819-830 (1998)

3. Ambrosetti, A., Rabinowitz, P.H.: Dual variational methods in critical point theory and applications. J. Funct. Anal. 14, 349-381 (1973)

4. Brezis, H., Nirenberg, L.: $H^{1}$ versus $C^{1}$ local minimizers. C. R. Acad. Sci. Paris, Sér. I Math. 317(5), 465-472 (1993)

5. Filippakis, M.E., Papageorgiou, N.S.: Multiple constant sign and nodal solutions for nonlinear elliptic equations with the $p$-Laplacian. J. Differ. Equ. 245(7), 1883-1922 (2008)

6. Fragnelli, G., Mugnai, D., Papageorgiou, N.S.: Positive and nodal solutions for parametric nonlinear Robin problems with indefinite potential. Discrete Contin. Dyn. Syst. 36(11), 6133-6166 (2016)

7. Fragnelli, G., Mugnai, D., Papageorgiou, N.S.: The Brezis-Oswald result for quasilinear Robin problems. Adv. Nonlinear Stud. 16(3), 603-622 (2016)

8. Gasiński, L., Papageorgiou, N.S.: Nonlinear Analysis. Series Mathematics Analysis Application, vol. 9. CRC Press, Boca Raton (2006)

9. Hu, S., Papageorgiou, N.S.: Handbook of Multivalued Analysis. Volume I Theory, vol. I. Kluwer Academic Publishers, Dordrecht (1997)

10. Hu, S., Papageorgiou, N.S.: Nonlinear Neumann equations driven by a nonhomogeneous differential operator. Commun. Pure Appl. Anal. 10(4), 1055-1078 (2011)

11. Hu, S., Papageorgiou, N.S.: Positive solutions for Robin problems with general potential and logistic reaction. Commun. Pure Appl. Anal. 15(6), 2489-2507 (2016)

12. Hu, S., Papageorgiou, N.S.: Solutions of nonlinear nonhomogeneous Neumann and Dirichlet problems. Commun. Pure Appl. Anal. 12(6), 2889-2922 (2013)

13. Lieberman, G.M.: Boundary regularity for solutions of degenerate elliptic equations. Nonlinear Anal. 12(11), 1203-1219 (1988)

14. Motreanu, D., Motreanu, V., Papageorgiou, N.S.: Topological and Variational Methods with Applications to Nonlinear Boundary Value Problems. Springer, New York (2014)

15. Mugnai, D.: Addendum to: multiplicity of critical points in presence of a linking: application to a superlinear boundary value problem. NoDEA. Nonlinear Differ. Equ. Appl. 11, 379-391 (2004). and a comment on the generalized Ambrosetti-Rabinowitz condition, NoDEA. Nonlinear Differ. Equ. Appl. 193, 299-301 (2012)

16. Mugnai, D., Papageorgiou, N.S.: Resonant nonlinear Neumann problems with indefinite weight. Ann. Sc. Norm. Super. Pisa Cl. Sci. (5) 11(4), 729-788 (2012)

17. Papageorgiou, N.S., Rădulescu, V.D.: Multiple solutions with precise sign for nonlinear parametric Robin problems. J. Differ. Equ. 256, 2449-2479 (2014)

18. Papageorgiou, N.S., Rădulescu, V.D.: Multiplicity of solutions for resonant Neumann problems with an indefinite and unbounded potential. Trans. Am. Math. Soc. 367(12), 8723-8756 (2015)

19. Papageorgiou, N.S., Rădulescu, V.D.: Nonlinear nonhomogeneous Robin problems with superlinear reaction term. Adv. Nonlinear. Stud. 16, 737-764 (2016)

20. Papageorgiou, N.S., Rădulescu, V.D., Repov̌̌, D.D.: Positive solutions for perturbations of the Robin eigenvalue problem plus an indefinite potential. Discrete Contin. Dyn. Syst. 37(5), 2589-2618 (2017)

Publisher's Note Springer Nature remains neutral with regard to jurisdictional claims in published maps and institutional affiliations. 